\title{
Dynamical Analysis and Simulation of a New Lorenz-Like Chaotic System
}

\author{
You Li, Ming Zhao $(D$, and Fengjie Geng \\ School of Science, China University of Geosciences (Beijing), Beijing 100083, China \\ Correspondence should be addressed to Ming Zhao; mingzhao@cugb.edu.cn
}

Received 5 November 2020; Revised 15 February 2021; Accepted 24 February 2021; Published 16 March 2021

Academic Editor: Abdul Qadeer Khan

Copyright (C) 2021 You Li et al. This is an open access article distributed under the Creative Commons Attribution License, which permits unrestricted use, distribution, and reproduction in any medium, provided the original work is properly cited.

\begin{abstract}
This work presents and investigates a new chaotic system with eight terms. By numerical simulation, the two-scroll chaotic attractor is found for some certain parameters. And, by theoretical analysis, we discuss the dynamical behavior of the new-type Lorenz-like chaotic system. Firstly, the local dynamical properties, such as the distribution and the local stability of all equilibrium points, the local stable and unstable manifolds, and the Hopf bifurcations, are all revealed as the parameters varying in the space of parameters. Secondly, by applying the way of Poincaré compactification in $\mathbb{R}^{3}$, the dynamics at infinity are clearly analyzed. Thirdly, combining the dynamics at finity and those at infinity, the global dynamical behaviors are formulated. Especially, we have proved the existence of the infinite heteroclinic orbits. Furthermore, all obtained theoretical results in this paper are further verified by numerical simulations.
\end{abstract}

\section{Introduction}

Chaos, as a magical and charming nonlinear phenomenon, has attracted attentions of many scholars in nonlinear dynamics community. This is not only because it is a mysterious and profound subject but also because it is beneficial to many practical applications. Especially, with the development of computer science, chaos has been applied to secure communication [1], biomedical system analysis [2], power system protection [3], and fluid mixing [4]. Accordingly, due to these influential applications, the synchronization and control of chaotic systems have fascinated numerous research studies; one may see [5-8] and references therein.

Ever since Lorenz discovered the chaotic phenomena in a simple 3D nonlinear ODE in 1963, more and more specialists devoted themselves to chaotic dynamics. Some of them were interested in finding different kinds of new chaotic models and discussed their dynamical properties, for example, Lü system [9], Chen system [10], Lorenz-type system [11-14], and other new chaotic system [15-19], while some of them focused their attentions on revisiting the existing chaotic system and explored some new phenomena which had not been found before [20-28]. All of these research studies are beneficial to reveal the essence of chaos.

As we know, if we add or change the linear or nonlinear term of existing chaotic system, then a new type chaotic system will be achieved. In [29], the authors constructed a new Lorenz-like chaotic system:

$$
\left\{\begin{array}{l}
\dot{x}=a(y-x), \\
\dot{y}=b x-l x z, \\
\dot{z}=-c z+h x^{2}+k y^{2} .
\end{array}\right.
$$

They pointed out that when the parameters satisfy $(a, b, l, c, h, k)=(10,40,1,2.5,2,2)$, this new system has a butterfly-shaped attractor.

Based on Lü system, Li and $\mathrm{Ou}$ [11] presented and considered a new chaotic system:

$$
\left\{\begin{array}{l}
\dot{x}=a(y-x), \\
\dot{y}=\mathrm{d} y-x z, \\
\dot{z}=-b z+f x^{2}+g x y,
\end{array}\right.
$$

where $a>0, b, d \in \mathbb{R}, g \geq 0, f \geq 0$, and $g+f>0$. The authors showed that when the parameters satisfy $a=10, d=6, b=3$, 
$f=1$, and $g=0$, system (2) will have a chaotic attractor. The local dynamical behavior, the existence for homoclinic orbits, and the existence for heteroclinic orbits of this new system were established.

After that, the authors [6] proposed the following new 3D chaotic system with a closed circle of the equilibrium points:

$$
\left\{\begin{array}{l}
\dot{x}=z, \\
\dot{y}=-z\left(x^{2}+a y-b y^{2}-c x z\right), \\
\dot{z}=(y-0.5|x|)^{2}+(0.15 x)^{2}-1,
\end{array}\right.
$$

where $a, b, c>0$. They studied the dynamics of system (3), and they designed an electronic circuit enforcement of this new system to illustrate its feasibility.

Recently, Wang and $\mathrm{Li}$ [30] replaced $x$ with $x^{2}$ in a Lorenz-type system and obtained a new nonlinear system as

$$
\left\{\begin{array}{l}
\dot{x}=a(y-x), \\
\dot{y}=c x^{2}-x^{2} z \\
\dot{z}=-b z+x^{2} y
\end{array}\right.
$$

where $a, b, c \in \mathbb{R}$ and $a \neq 0$. Although the chaotic attractor has not been found so far, they proved that system (4) has a single heteroclinic trajectory, which is the new character of the proposed system.

Motivated by the results about the different new nonlinear systems, in this paper, by adding a linear term in system (2), we propose a new system:

$$
\left\{\begin{array}{l}
\dot{x}=a(y-x), \\
\dot{y}=-\mathrm{d} x+c y-x z, \\
\dot{z}=-b z+f x^{2}+g x y,
\end{array}\right.
$$

where $a>0, b, c, d \in \mathbb{R}, g \geq 0, f \geq 0$, and $g+f>0$. Notice that there are eight terms in this system, and it is obvious that it is not topologically equivalent to any form of the generalized Lorenz system. Furthermore, Lü system [9] and system (2) are included in our new system.

By taking $(a, d, c, b, f, g)=(10,2,6,3,2,1,1)$ and $t=5000$, we can achieve the Lyapunov exponents of system (5) as $\lambda_{\mathrm{LE}_{1}}=0.2603, \lambda_{\mathrm{LE}_{2}}=-0.0008$, and $\lambda_{\mathrm{LE}_{3}}=-7.2595$, which means that there is a chaotic phenomena in system (5). And, the numerical simulation in Figure 1 shows that system (5) has a two-scroll chaotic attractor which looks like Lorenz attractor. Hence, this new system can be called as Lorenz-like system. Due to the above discovery, we are inspired to explore some more and new complex dynamical behaviors of system (5).

Therefore, in this paper, our main purpose is to explore the global dynamics of system (5) in detail. It is worthy pointing out that the dynamics at infinity especially the existence of infinite heteroclinic orbits have not been mentioned in [11].

This paper is constructed as follows. In Section 2, the basic dynamics of system (5), including the existence and their local properties of the equilibrium points, are provided. Particularly, the specific expressions of local stable (resp. unstable) manifolds and the corresponding numerical simulations are also presented. In Section 3, by virtue of the Poincaré compactification in $\mathbb{R}^{3}$, we present the dynamical behaviors on the sphere at infinity, where we find the infinite heteroclinic orbits for some certain parameters. In Section 4, we give a brief conclusion and some future work.

\section{Local Dynamical Behavior of System (5)}

In the following, we consider the local dynamical properties for system (5), including the existence and the stability of all equilibrium points, Hopf bifurcation, and the local structure of trajectories.

First, we indicate that system (5) satisfies the following properties:

(1) Symmetry and invariance.

(2) Normality.

(3) Dissipativity.

These can be easily proved with similar arguments to [11], so we omit it here.

2.1. The Existence of Equilibrium Points. In order to discuss the existence of equilibrium points for system (5), we need to solve the algebraic equations as follows:

$$
\begin{aligned}
a(y-x) & =0, \\
-\mathrm{d} x+c y-x z & =0, \\
-b z+f x^{2}+g x y & =0 .
\end{aligned}
$$

By some simple computations, we achieve the following results.

Theorem 1. For system (5), we have the following true statements:

(1) If $b(c-d)<0$ or $c-d=0$, then there exists exactly one equilibrium point $E_{0}$.

(2) If $b=0$, then there exist nonisolated equilibrium points $E_{z}$ for $z \in R$.

(3) If $b(c-d)>0$, then there exist three equilibrium points $E_{0}$ and $E_{ \pm}$.

Here, $\quad E_{0}=(0,0,0), \quad E_{z}=(0,0, z), \quad$ and $E_{ \pm}=( \pm \sqrt{b(c-d) /(g+f)}, \pm \sqrt{b(c-d) /(g+f)}, c-d)$.

2.2. Local Dynamical Properties of the Equilibrium Point $E_{0}$. For the equilibrium point $E_{0}$ of system (5), we can get its Jacobian matrix $A_{0}$ :

$$
A_{0}=\left(\begin{array}{ccc}
-a & a & 0 \\
-d & c & 0 \\
0 & 0 & -b
\end{array}\right)
$$

Then, the characteristic equation of $A_{0}$ is obtained:

$$
p(\lambda)=(\lambda+b)\left[\lambda^{2}+(a-c) \lambda-a(c-d)\right]=0 .
$$



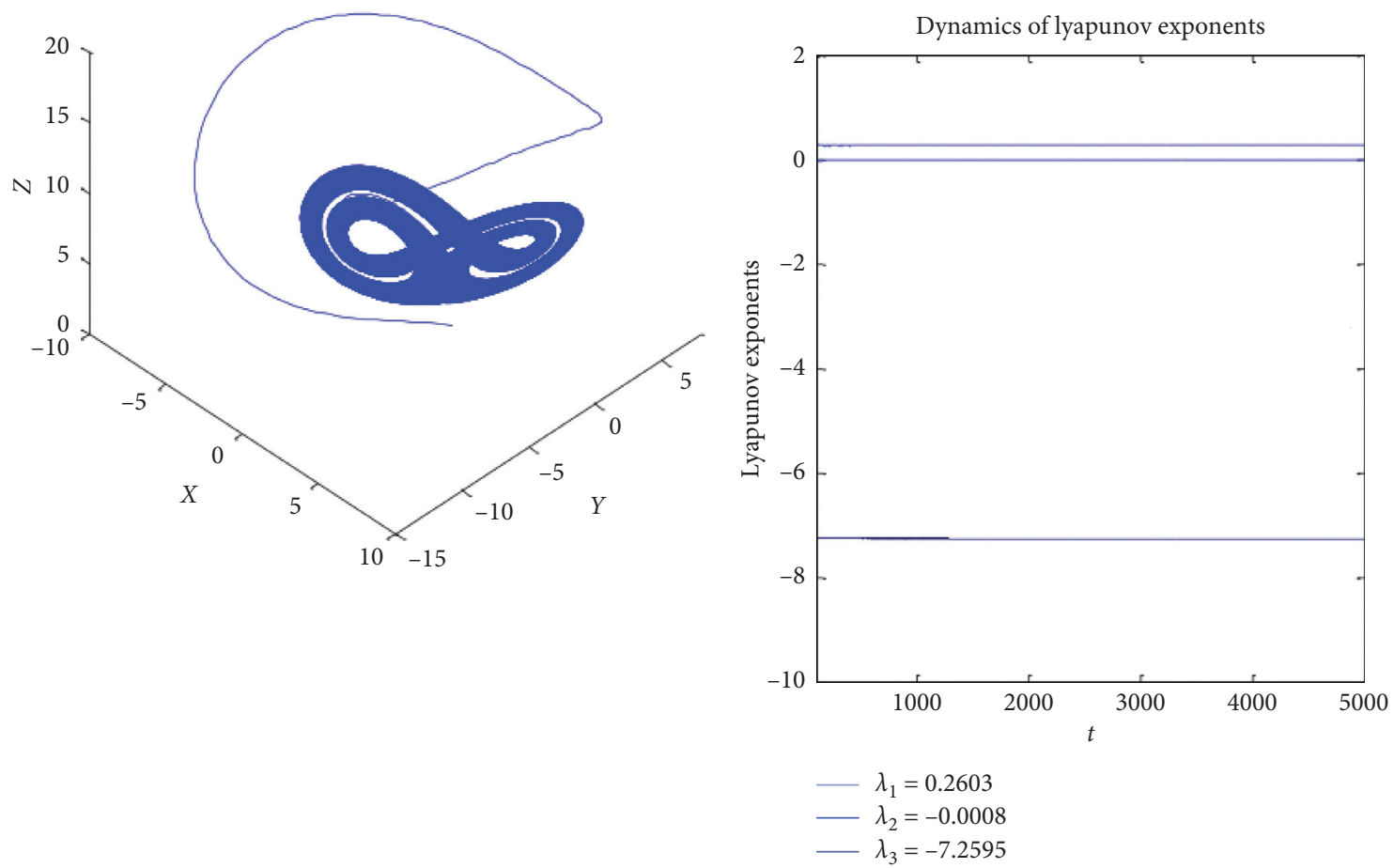

(a)

(b)

Figure 1: (a-b) Phase portrait and Lyapunov exponents for system (5) when $(a, d, c, b, f, g)=(10,2,6,3,2,1,1)$.

We will discuss the local dynamical properties of equilibrium point $E_{0}$ in the following three sections.

2.2.1. The Case $c-d>0$. Due to equation (8), we obtain three eigenvalues:

$$
\begin{aligned}
& \lambda_{1,2}=\frac{-(a-c) \pm \sqrt{(c-a)^{2}+4 a(c-d)}}{2}, \\
& \lambda_{3}=-b .
\end{aligned}
$$

Their corresponding eigenvectors are

$$
\begin{aligned}
v_{1,2} & =\left(\frac{-(a+c) \pm \sqrt{\Delta}}{-2 d}, 1,0\right), \\
v_{3} & =(0,0,1),
\end{aligned}
$$

where $\Delta=(a-c)^{2}+4 a(c-d)$. It is not difficult to see that $\lambda_{1,2}$ are both real roots that satisfy $\lambda_{1}>0$ and $\lambda_{2}<0$. The root $\lambda_{3}>0$ (resp. $\left.\lambda_{3}<0\right)$ for $b<0$ (resp. $b>0$ ).

Applying the center manifold theorem, we can derive the stability of $E_{0}$.

Lemma 1. For $c-d>0$ and $b<0, E_{0}$ is a saddle. And, it has a $2 D$ unstable manifold that embraces $z$-axis and a $1 D$ stable manifold $W_{\text {loc }}^{s}$, which can be expressed by

$$
W_{\mathrm{loc}}^{s}=\left\{\left(\begin{array}{c}
x \\
y \\
z
\end{array}\right) \begin{array}{c}
|x| \ll 1 \\
z=\frac{a+c-\sqrt{\Delta}}{2 a} x+O\left(x^{2}\right), \\
z=\frac{2 a f(b+c-a+\sqrt{\Delta})+g(a+c-\sqrt{\Delta})(b+c-a+\sqrt{\Delta})}{2 a b(b-2 a+2 c)-8 a^{2}(c-d)} x^{2}+O\left(x^{3}\right) .
\end{array}\right\} .
$$


The corresponding illustrative phase portraits are shown in Figure 2.

$$
W_{\mathrm{loc}}^{s}=\left\{\left(\begin{array}{l}
x \\
y \\
z
\end{array}\right) \begin{array}{l}
|x| \ll 1 \\
\mid y=H(x) A_{1}\left[\begin{array}{c}
1 \\
z=K(x)
\end{array}\right]=\lambda_{2}\left[\begin{array}{c}
1 \\
H^{\prime}(0)
\end{array}\right], H(0)=K(0)=0
\end{array}\right\},
$$

where $A_{1}=\left[\begin{array}{ll}-a & a \\ -d & c\end{array}\right]$

Let $\quad y=H(x)=h_{1} x+h_{2} x^{2}+O\left(x^{3}\right) \quad$ and $z=K(x)=k_{1} x+k_{2} x^{2}+O\left(x^{3}\right)$. Put them into system (5) and then compare the coefficients of the same term on both sides, and we achieve

$$
\left\{\begin{aligned}
\left(a h_{1}-a-c\right) h_{1}+d & =0, \\
\left(3 a h_{1}-2 a-c\right) h_{2} & =-k_{1}, \\
a\left(h_{1}-1\right) k_{1} & =-b k_{1}, \\
a\left[k_{1} h_{2}+2 k_{2}\left(h_{1}-1\right)\right] & =-b k_{2}+f+g h_{1} .
\end{aligned}\right.
$$

It follows from the matrix equation,

$$
\left[\begin{array}{ll}
-a & a \\
-d & c
\end{array}\right]\left[\begin{array}{c}
1 \\
H^{\prime}(0)
\end{array}\right]=\lambda_{2}\left[\begin{array}{c}
1 \\
H^{\prime}(0)
\end{array}\right]
$$

Proof. When $c-d>0$ and $b<0$, it is obvious that $\lambda_{1}>0$, $\lambda_{3}>0$, and $\lambda_{2}<0$. This means that $E_{0}$ is a saddle, which consists of a 2D unstable manifold that includes $z$-axis and a $1 \mathrm{D}$ stable manifold $W_{\text {loc }}^{s}$, which can be expressed by that $h_{1}=(a+c-\sqrt{\Delta} / 2 a)$. So, we achieve

$$
\begin{aligned}
& k_{1}=0, \\
& k_{2}=\frac{2 a f(b+c-a+\sqrt{\Delta})+g(c+a-\sqrt{\Delta})(b+c-a+\sqrt{\Delta})}{2 a b(b-2 a+2 c)-8 a^{2}(c-d)}, \\
& h_{2}=0 .
\end{aligned}
$$

The proof of Lemma 1 is then finished.

Lemma 2. For $c-d>0$ and $b>0, E_{0}$ is a saddle. And, it has a $2 \mathrm{D}$ stable manifold that embraces $z$-axis and a $1 \mathrm{D}$ unstable manifold $W_{\text {loc }}^{u}$, which is expressed as

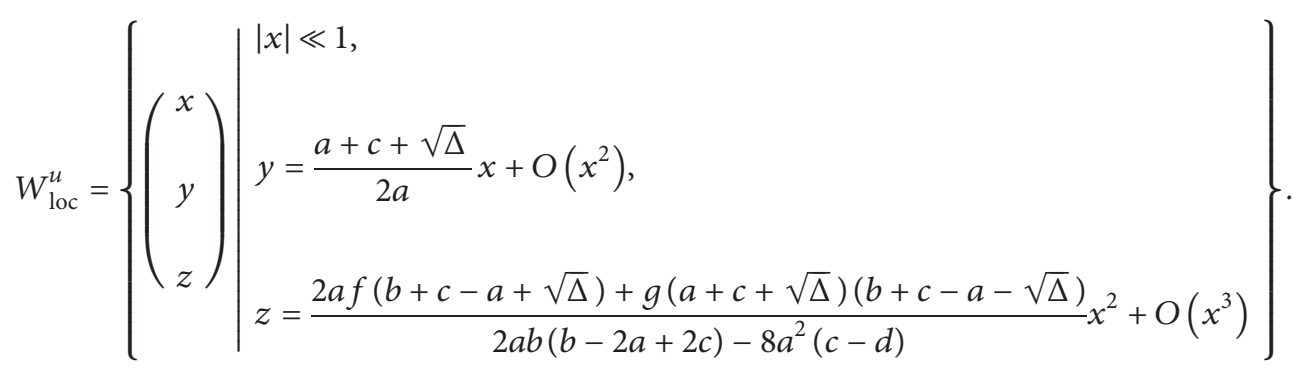

Proof. When $c-d>0$ and $b>0$, it is obvious that $\lambda_{2}<0$, $\lambda_{3}<0$, and $\lambda_{1}>0$. Therefore, $E_{0}$ is a saddle, which consists of a 2D stable manifold that includes $z$-axis, and a 1D unstable manifold $W_{\text {loc }}^{u}$, which is written as

$$
\left.W_{\mathrm{loc}}^{u}=\left\{\left(\begin{array}{l}
x \\
y \\
z
\end{array}\right) \begin{array}{c}
|x| \ll 1 \\
\mid y=H(x) A_{2}\left[\begin{array}{c}
1 \\
z=K(x)
\end{array}\right. \\
H^{\prime}(0)
\end{array}\right]=\lambda_{1}\left[\begin{array}{c}
1 \\
H^{\prime}(0)
\end{array}\right], H(0)=K(0)=0\right\},
$$


where $A_{2}=\left[\begin{array}{ll}-a & a \\ -d & c\end{array}\right]$

Let $y=H(x)=h_{1} x+h_{2} x^{2}+O\left(x^{3}\right)$ and $z=K(x)=k_{1}$ $(x)+k_{2} x^{2}+O\left(x^{3}\right)$. Similar to Lemma 1 , we may derive that

$h_{1}=\frac{a+c+\sqrt{\Delta}}{2 a}$,

$h_{2}=0$,

$k_{1}=0$,

$k_{2}=\frac{2 a f(b+c-a+\sqrt{\Delta})+g(c+a+\sqrt{\Delta})(b+c-a-\sqrt{\Delta})}{2 a b(b-2 a+2 c)-8 a^{2}(c-d)}$.

Thus, we complete the proof of Lemma 2.

2.2.2. The Case $c-d=0$

Lemma 3. Suppose $c-d=0$, then we have the following true statements:

(1) If $d-a<0$, then

(i) For $b>0, E_{0}$ is a nonhyperbolic point and asymptotically stable. The corresponding illustrative phase portrait is depicted in Figure 3.

(ii) For $b<0, E_{0}$ is a nonhyperbolic point, and it is of saddle-center type.

(2) If $d-a>0$, then

(i) For $b>0, E_{0}$ is a nonhyperbolic point, and it is of saddle-center type.

(ii) For $b<0, E_{0}$ is a nonhyperbolic point and unstable.

Proof. (1) When $c-d=0, d<a$, and $b>0, E_{0}$ has three eigenvalues $\lambda_{1}<0, \lambda_{3}<0$, and $\lambda_{2}=0$. Thus, it is nonhyperbolic. In the following, we will use the center manifold theory to further determine its stability.

Let

$$
\left[\begin{array}{l}
x \\
y \\
z
\end{array}\right]=\left[\begin{array}{ccc}
1 & -a & 0 \\
1 & -d & 0 \\
0 & 0 & 1
\end{array}\right]\left[\begin{array}{l}
x_{1} \\
y_{1} \\
z_{1}
\end{array}\right] .
$$

Then, system (5) turns into

$$
\left[\begin{array}{c}
\dot{x}_{1} \\
\dot{y}_{1} \\
\dot{z}_{1}
\end{array}\right]=\left[\begin{array}{ccc}
0 & 0 & 0 \\
0 & -(a-d) & 0 \\
0 & 0 & -b
\end{array}\right]\left[\begin{array}{c}
x_{1} \\
y_{1} \\
z_{1}
\end{array}\right]+\left[\begin{array}{c}
-\frac{a}{a-d}\left(x_{1}-a y_{1}\right) z_{1} \\
-\frac{1}{a-d}\left(x_{1}-a y_{1}\right) z_{1} \\
f\left(x_{1}-a y_{1}\right)^{2}+g\left(x_{1}-a y_{1}\right)\left(x_{1}-\mathrm{d} y_{1}\right)
\end{array}\right] .
$$

Based on the center manifold theory, we can determine the stability of the equilibrium point $E_{0}$ by investigating a first-order ODE restrict to its center manifold, and the center manifold can be expressed as

$$
\begin{aligned}
W_{\text {loc }}^{c} & =\left\{\left(x_{1}, y_{1}, z_{1}\right) \in R^{3} \mid y_{1}=h_{1}\left(x_{1}\right), z_{1}\right. \\
& =h_{2}\left(x_{1}\right),\left|x_{1}\right|<\delta, h_{1}(0)=h_{2}(0) \\
& \left.=h_{1}^{\prime}(0)=h_{2}^{\prime}(0)=0\right\},
\end{aligned}
$$

with $\delta$ sufficiently small.

Assume $\quad h_{1}\left(x_{1}\right)=\alpha_{1} x_{1}^{2}+\beta_{1} x_{1}^{3}+O\left(x^{4}\right) \quad$ and $h_{2}\left(x_{1}\right)=\alpha_{2} x_{1}^{2}+\beta_{2} x_{1}^{3}+O\left(x^{4}\right)$; then, it must satisfy

$$
\left\{\begin{array}{l}
\frac{a}{a-d} h_{1}^{\prime}\left(x_{1}\right)\left(x_{1}-a h_{1}\left(x_{1}\right)\right) h_{2}\left(x_{1}\right)-(a-d) h_{1}\left(x_{1}\right)-\frac{1}{a-d}\left(x_{1}-a h_{1}\left(x_{1}\right)\right) h_{2}\left(x_{1}\right)=0 \\
\frac{a}{a-d} h_{2}^{\prime}\left(x_{1}\right)\left(x_{1}-a h_{1}\left(x_{1}\right)\right) h_{2}\left(x_{1}\right)-b h_{2}\left(x_{1}\right)+f\left(x_{1}-a h_{1}\left(x_{1}\right)\right)^{2}+g\left(x_{1}-a h_{1}\left(x_{1}\right)\right)\left(x_{1}-d h_{1}\left(x_{1}\right)\right)=0 .
\end{array}\right.
$$

By some computations, we obtain 


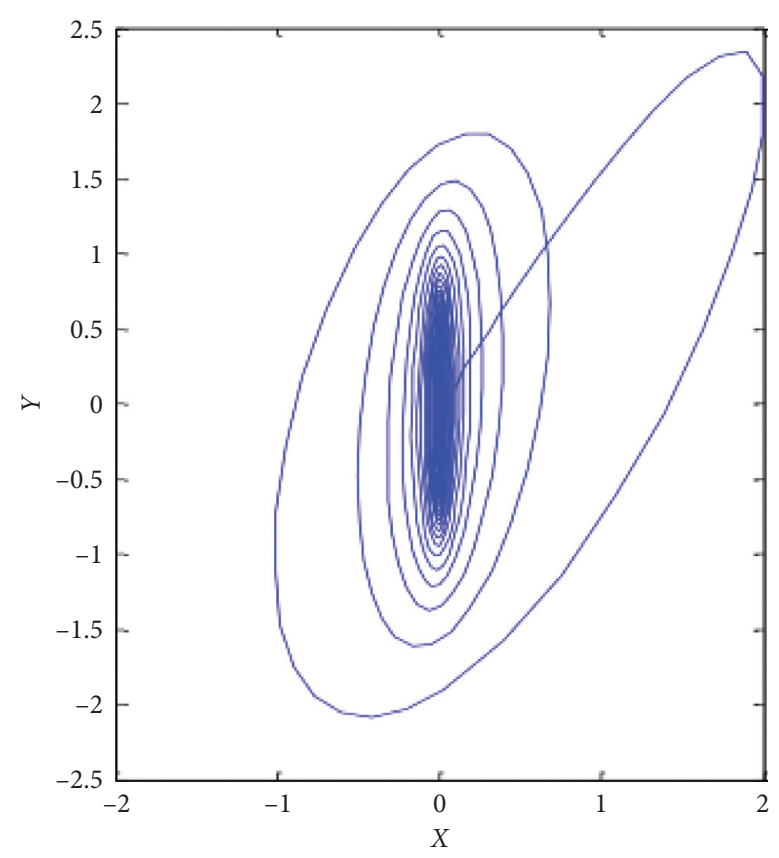

(a)

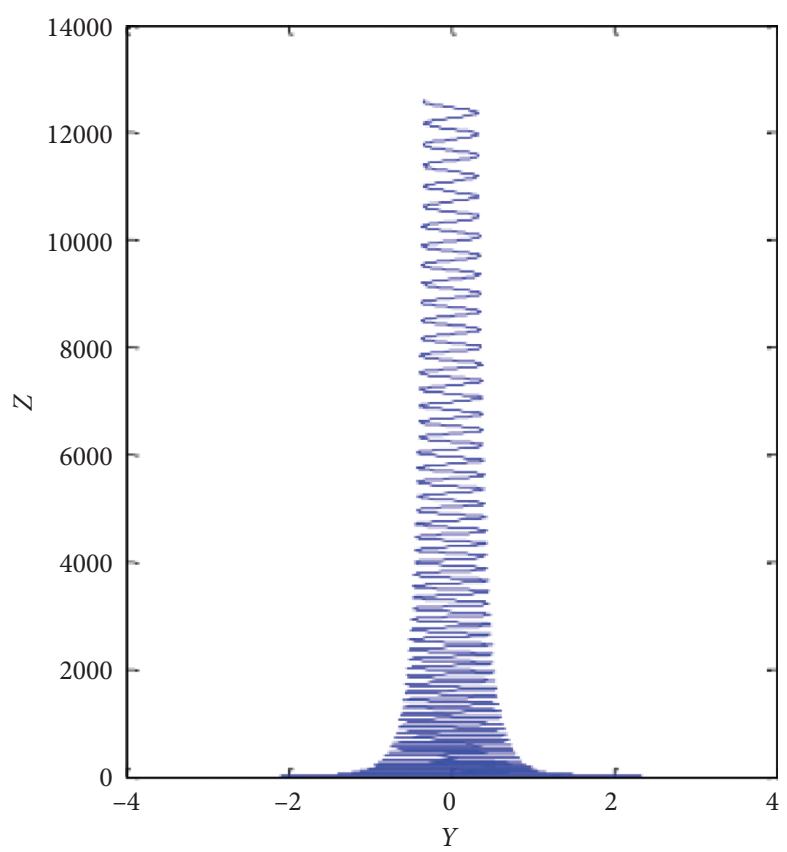

(c)

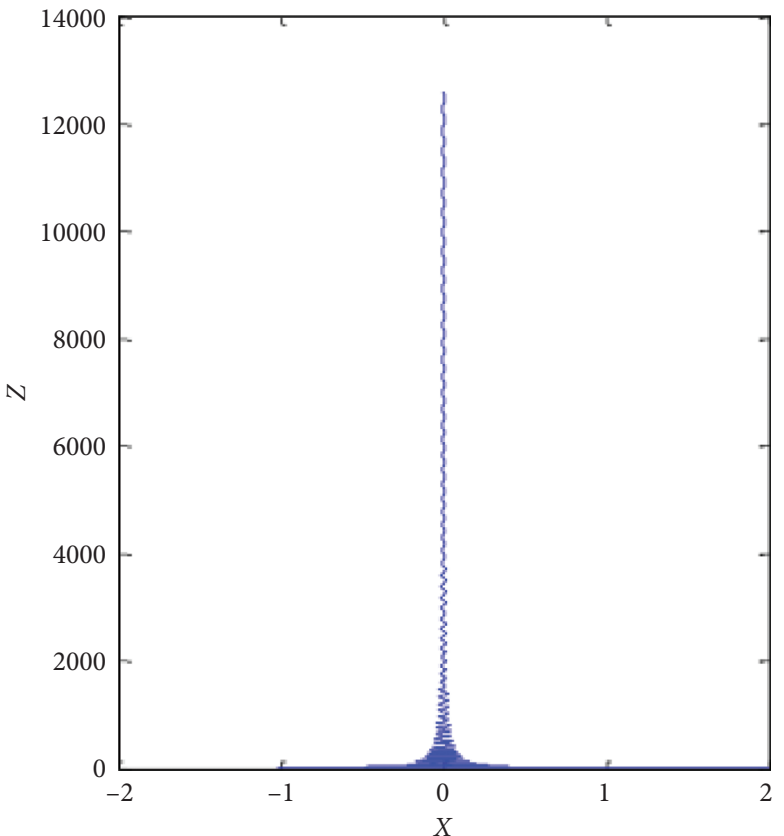

(b)

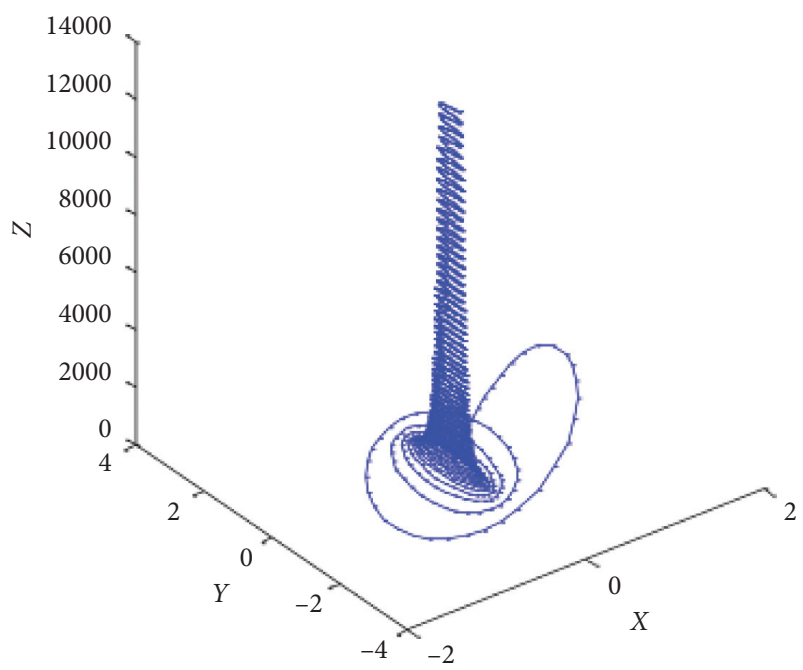

(d)

Figure 2: (a-d) Phase portraits for system (5) when $(a, b, c, d, f, g)=(3,-1,2,0,1,0)$ : (a) in the $x y$-plane; (b) in the $x z$-plane; (c) in the $y z$-plane; (d) in $R^{3}$.

$$
\left\{\begin{array}{l}
h_{1}\left(x_{1}\right)=-\frac{f+g}{b(a-d)^{2}} x_{1}^{3}+O\left(x_{1}^{4}\right), \\
h_{2}\left(x_{1}\right)=\frac{f+g}{b} x_{1}^{2}+O\left(x_{1}^{4}\right) .
\end{array}\right.
$$

Hence, system (5) restricted to the center manifold may be written as

$$
\dot{x}_{1}=-\frac{a(f+g)}{b(a-d)} x_{1}^{3}+O\left(x_{1}^{4}\right) .
$$

It then follows that the equilibrium point $E_{0}$ is asymptotically stable.

For $c-d=0, d<a$, and $b<0, E_{0}$ has three eigenvalues $\lambda_{1}<0, \lambda_{3}>0$, and $\lambda_{2}=0$, which implies $E_{0}$ is nonhyperbolic point and is of saddle-center type. Hence, we finish the proof of Lemma 3 (1). 


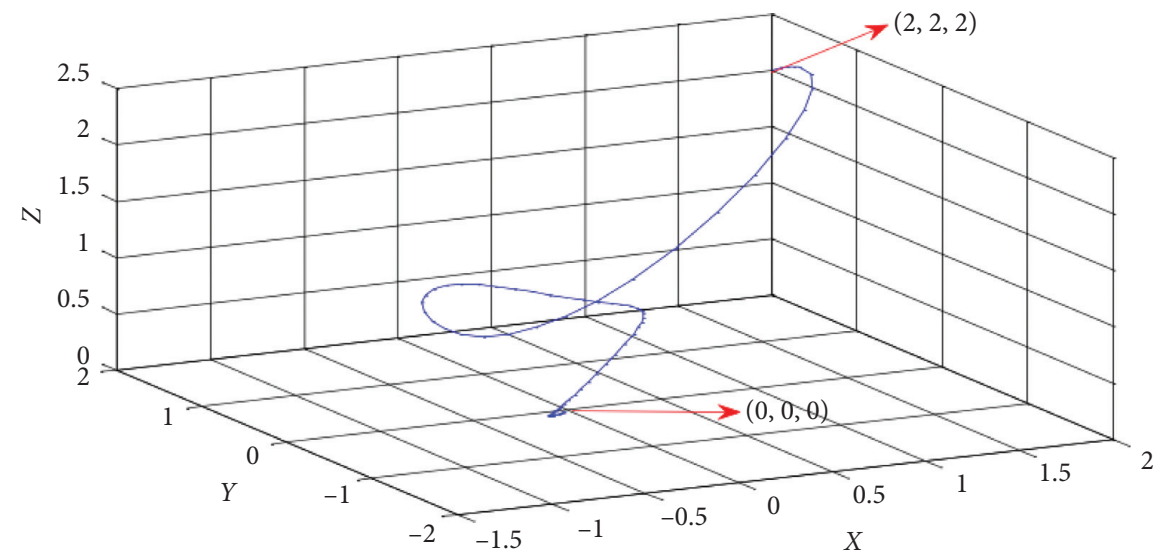

FIgURE 3: Phase portrait for system (5) when the parameters satisfy $(a, b, c, d, f, g)=(3,1,2,2,1,0)$ in $R^{3}$. Obviously, $E_{0}$ is asymptotically stable.

(2) When $c-d=0, d>a$, and $b>0$, the equilibrium point $E_{0}$ has three eigenvalues $\lambda_{1}>0, \lambda_{3}<0$, and $\lambda_{2}=0$, which means $E_{0}$ is nonhyperbolic point and is of saddle-center type.

When $c-d=0, d>a$, and $b<0$, the equilibrium point $E_{0}$ has three eigenvalues $\lambda_{1}>0, \lambda_{3}>0$, and $\lambda_{2}=0$. So, $E_{0}$ is nonhyperbolic point and unstable. Hence, we finish the proof of Lemma 3 (2).

Remark 1. If $d=a$ and $c-d=0$, system (5) will have a center manifold with high-order terms.

2.2.3. The Case $c-d<0$. When $c-d<0$, the dynamical properties of the equilibrium point $E_{0}$ are formulated in Table 1.

Especially, when $c-d<0, b>0$, and $c-a=0$, we have the lemma as follows.

Lemma 4. Suppose $c-d<0, c-a=0$, and $b>0$, then the Hopf bifurcation will happen to system (5) at the equilibrium point $E_{0}$, and there exists an orbit with the period $T=(\pi / \sqrt{a(d-c)})$. The corresponding illustrative phase portrait is shown in Figure 4.

Proof. When $c-d<0, b>0$, and $c-a=0$, the characteristic equation (8) has roots $\lambda_{3}=-b<0$ and $\lambda_{1,2}= \pm 2 \sqrt{a(d-c)}$. Then, we can obtain

$$
\left.\frac{\mathrm{dRe}\left(\lambda_{1}\right)}{\mathrm{d} c}\right|_{c=a}=\frac{1}{2}>0
$$

By Hopf bifurcation theory [31], a Hopf bifurcation will happen to system (5) at $E_{0}$. This means a limit cycle will occur at $c=a$, which has initial period $T=(\pi / \sqrt{a(d-c)})$ and increases with $c$. So, the proof of Lemma 4 is then completed.

Summing up the above discussions, we get the main theorem as follows.
Theorem 2. Assume $a>0, g \geq 0, f \geq 0, g+f>0$, and $b, c, d \in \mathbb{R}$, then the equilibrium point $E_{0}$ of system (5) has the local dynamical properties which are given in Table 2.

2.3. Local Dynamic Behavior of the Nonisolated Equilibrium Points $E_{z}$. The characteristic equation at the nonisolated points $E_{z}$ of system (5) is

$$
\lambda\left[\lambda^{2}+(a-c) \lambda-a(c-d-z)\right]=0 .
$$

By analyzing the characteristic roots of the above equation, we can get the results as follows.

Theorem 3. Suppose that $a>0, b=0, c, d \in \mathbb{R}, g \geq 0, f \geq 0$, and $g+f>0$, then the equilibrium points $E_{z}$ have the local dynamical properties which are summed in Table 3.

2.4. Local Dynamical Behavior of the Equilibrium Points $E_{ \pm}$. In what follows, we deal with the dynamical behavior of $E_{ \pm}$, which means $b(c-d)>0$. By symmetry of (5), we know that the stability of $E_{-}$can be achieved from that of the equilibrium point $E_{+}$. Thus, we just consider the local dynamical behavior of $E_{+}$.

For convenience in our later discussion, we first give some results of the polynomial with real coefficient:

$$
p(\lambda)=\lambda^{3}+a_{1} \lambda^{2}+a_{2} \lambda+a_{3} .
$$

Set three roots of equation $p(\lambda)=0$ be $\lambda_{1}, \lambda_{2}$, and $\lambda_{3}$. Then, it is easy to obtain

$$
\left\{\begin{array}{l}
\lambda_{1}+\lambda_{2}+\lambda_{3}=-a_{1}, \\
\lambda_{1} \lambda_{2}+\lambda_{2} \lambda_{3}+\lambda_{1} \lambda_{3}=a_{2}, \\
\lambda_{1} \lambda_{2} \lambda_{3}=-a_{3} .
\end{array}\right.
$$

Let

$$
H=\left[\begin{array}{ccc}
a_{1} & a_{3} & 0 \\
1 & a_{2} & 0 \\
0 & a_{1} & a_{3}
\end{array}\right] .
$$


TABLE 1: The properties of equilibrium point $E_{0}$ for $c-d<0$.

\begin{tabular}{lccc}
\hline Conditions & & Eigenvalues & Type \\
\hline \multirow{2}{*}{$c-a>0$} & $b>0$ & $\operatorname{Re}\left(\lambda_{1,2}\right)>0, \lambda_{3}<0$ & Saddle-focus \\
& $b<0$ & $\operatorname{Re}\left(\lambda_{1,2}\right)>0, \lambda_{3}>0$ & Unstable node-focus \\
\hline \multirow{2}{*}{$c-a=0$} & $b>0$ & $\lambda_{1,2}= \pm 2 \sqrt{a(d-c)} i, \lambda_{3}<0$ & Non-hyperbolic \\
& $b<0$ & $\lambda_{1,2}= \pm 2 \sqrt{a(d-c)} i, \lambda_{3}>0$ & Non-hyperbolic \\
\multirow{2}{*}{$c-a<0$} & $b>0$ & $\operatorname{Re}\left(\lambda_{1,2}\right)<0, \lambda_{3}<0$ & Stable node-focus \\
& $b<0$ & $\operatorname{Re}\left(\lambda_{1,2}\right)<0, \lambda_{3}>0$ & Saddle-focus \\
\hline
\end{tabular}

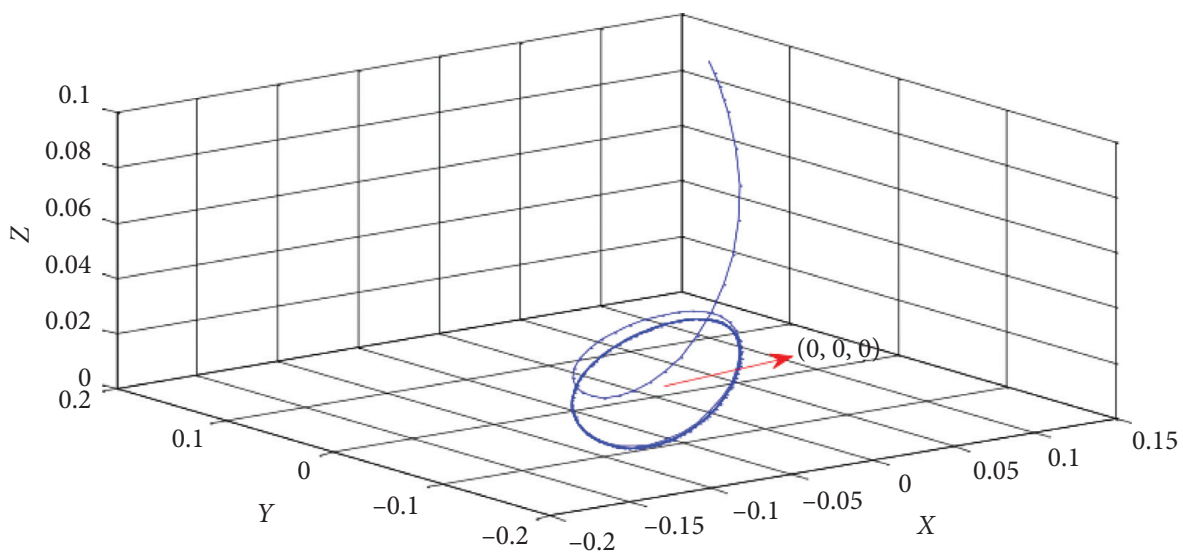

Figure 4: Phase portrait for system (5) when $(a, b, c, d, f, g)=(3,1,3,4,1,0)$.

We can obtain the principal minors for $H$ which are $H_{1}=a_{1}, H_{2}=a_{1} a_{2}-a_{3}$, and $H_{3}=a_{3} H_{2}$. Thus, we have the following results.

Lemma 5. Suppose $a_{3}>0$, then we have the following true statements:

(1) $p(\lambda)=0$ has no root $\lambda_{i}=0(i=1,2,3)$.

(2) If $p(\lambda)=0$ has a real root and a pair of pure imaginary roots, then $\mathrm{H}_{2}=0$.

(3) If one of $H_{i}$ satisfies $H_{i}<0$, then $p(\lambda)=0$ has at least a root $\lambda_{i}$ with $\operatorname{Re}\left(\lambda_{i}\right)>0(i=1,2,3)$.

Proof.

(1) It follows from $a_{3}=-\lambda_{1} \lambda_{2} \lambda_{3}>0$ that $\lambda_{i} \neq 0$ ( $i=$ $1,2,3)$. So, we finish the proof of (1).

(2) When $a_{3}>0$, suppose that $p(\lambda)=0$ has a real root $\lambda_{1}$ and a pair of pure imaginary roots $\lambda_{2,3}= \pm \omega i(\omega>0)$. It follows from (1) that $\lambda_{1} \neq 0$; then, from (11), we can derive $H_{2}=a_{1} a_{2}-a_{3}=0$, completing the proof of (2).

(3) Assume $a_{3}>0$, when $p(\lambda)=0$ has three real roots or a pair of conjugate roots with nonzero real parts and a real root; then, it follows from the Routh-Hurwitz criterion that $p(\lambda)=0$ has at least a root $\lambda_{i}$ with $\operatorname{Re}\left(\lambda_{i}\right)>0(i=1,2,3)$. And, when $p(\lambda)=0$ has a real root $\lambda_{1}$ and a pair of pure imaginary roots $\lambda_{2,3}$, if $H_{2}<0$, then according to (2), we know $H_{2}=0$, a contradiction; if $\mathrm{H}_{3}<0$, then from $\mathrm{H}_{3}=a_{3} \mathrm{H}_{2}$ and
TABLE 2: Local dynamical properties of equilibrium point $E_{0}$.

\begin{tabular}{|c|c|c|c|c|}
\hline $\begin{array}{l}\text { Cond } \\
c-d\end{array}$ & $\begin{array}{l}\text { tions } \\
d-a\end{array}$ & $c-a$ & $b$ & Property \\
\hline$>0$ & & & & $\begin{array}{l}\text { A } 2 \mathrm{D} W_{\mathrm{loc}}^{s} \text { and a } 1 \mathrm{D} W_{\mathrm{loc}}^{u} \\
\mathrm{~A} 1 \mathrm{D} W_{\mathrm{loc}}^{s} \text { and a } 2 \mathrm{D} W_{\mathrm{loc}}^{u}\end{array}$ \\
\hline$=0$ & $=0$ & & $\begin{array}{l}>0 \\
<0 \\
>0 \\
<0 \\
>0 \\
<0\end{array}$ & $\begin{array}{l}\text { A } 1 \mathrm{D} W_{\text {loc }}^{s}, \text { a } 1 \mathrm{D} W_{\text {loc }}^{u} \text {, and a } 1 \mathrm{D} W_{\text {loc }}^{c} \\
\text { A } 2 \mathrm{D} W_{\text {loc }}^{u} \text { and a } 1 \mathrm{D} W_{\text {loc }}^{c} \\
\text { A } 1 \mathrm{D} W_{\text {loc }}^{s} \text { and a } 2 \mathrm{D} W_{\text {loc }}^{c} \\
\text { A } 1 \mathrm{D} W_{\text {loc }}^{u} \text { and a } 2 \mathrm{D} W_{\text {loc }}^{c} \\
\text { A } 2 \mathrm{D} W_{\text {loc }}^{s} \text { and a } 1 \mathrm{D} W_{\text {loc }}^{c} \\
\text { A 1D } W_{\text {loc }}^{s}, \text { a } 1 \mathrm{D} W_{\text {loc }}^{u} \text {, and a } 1 \mathrm{D} W_{\text {loc }}^{c}\end{array}$ \\
\hline$<0$ & & $\begin{array}{l}=0 \\
<0\end{array}$ & $\begin{array}{l}>0 \\
<0 \\
>0 \\
<0 \\
>0 \\
<0\end{array}$ & $\begin{array}{c}\text { A } 1 \mathrm{D} W_{\text {loc }}^{s} \text { and a } 2 \mathrm{D} W_{\text {loc }}^{u} \\
\mathrm{~A} 3 \mathrm{D} W_{\text {loc }}^{u} \\
\text { A } 1 \mathrm{D} W_{\text {loc }}^{s} \text { and a } 2 \mathrm{D} W_{\text {loc }}^{c} \\
\text { A } 1 \mathrm{D} W_{\text {loc }}^{u} \text { and a } 2 \mathrm{D} W_{\text {loc }}^{c} \\
\text { A 3D } W_{\text {loc }}^{s} \\
\text { A 2D } W_{\text {loc }}^{s} \text { and a } 1 \mathrm{D} W_{\text {loc }}^{u}\end{array}$ \\
\hline
\end{tabular}

TABLE 3: Local dynamical properties of the nonisolated equilibrium points $E_{z}$.

\begin{tabular}{|c|c|c|}
\hline Conditions & & Property \\
\hline$c-a>0$ & $\begin{array}{l}z>c-d \\
z=c-d \\
z<c-d\end{array}$ & $\begin{array}{c}\text { A } 1 \mathrm{D} W_{\mathrm{loc}}^{s}, \text { a } 1 \mathrm{D} W_{\mathrm{loc}}^{u} \text {, and a } 1 \mathrm{D} W_{\mathrm{loc}}^{c} \\
\text { A } 1 \mathrm{D} W_{\text {loc }}^{u} \text { and a } 2 \mathrm{D} W_{\text {loc }}^{c} \\
\text { A } 2 \mathrm{D} W_{\text {loc }}^{u} \text { and a } 1 \mathrm{D} W_{\mathrm{loc}}^{c}\end{array}$ \\
\hline$c-a=0$ & $\begin{array}{l}z>c-d \\
z=c-d \\
z<c-d\end{array}$ & $\begin{array}{l}\text { May be fold-Hopf bifurcation } \\
\text { A } 3 \mathrm{D} W_{\text {loc }}^{c} \\
\text { A } 1 \mathrm{D} W_{\mathrm{loc}}^{s} \text {, a } 1 \mathrm{D} W_{\mathrm{loc}}^{u} \text {, and a } 1 \mathrm{D} W_{\text {loc }}^{c}\end{array}$ \\
\hline$c-a<0$ & $\begin{array}{l}z>c-d \\
z=c-d \\
z<c-d\end{array}$ & $\begin{array}{c}\text { A } 2 \mathrm{D} W_{\text {loc }}^{s} \text { and a } 1 \mathrm{D} W_{\text {loc }}^{c} \\
\text { A } 1 \mathrm{D} W_{\text {loc }}^{s} \text { and a } 2 \mathrm{D} W_{\text {loc }}^{c} \\
\text { A } 1 \mathrm{D} W_{\text {loc }}^{s} \text {, a } 1 \mathrm{D} W_{\text {loc }}^{u} \text {, and a } 1 \mathrm{D} W_{\text {loc }}^{c}\end{array}$ \\
\hline
\end{tabular}


$a_{3}>0$, we can get $H_{2}<0$, this is also a contradiction; if $H_{1}<0$, from the first equation of (12), we obtain $\lambda_{1}=\lambda_{1}+\lambda_{2}+\lambda_{3}=-H_{1}>0$. So, if one of $H_{i}(i=1,2,3)$ satisfies $H_{i}<0$, then $p(\lambda)=0$ has at least a root $\lambda_{i}$ with positive real part. Thus, (3) is proved.

The Jacobian matrix $A$ at $E_{+}$of system (5) is

$$
A=\left[\begin{array}{ccc}
-a & a & 0 \\
-c & c & -\Lambda \\
(2 f+g) \Lambda & g \Lambda & -b
\end{array}\right]
$$

where $\Lambda=\sqrt{b(c-d) /(f+g)}$. And, its characteristic equation is

$$
\begin{aligned}
k(\lambda)= & \lambda^{3}+(a+b-c) \lambda^{2}+b\left(a-\frac{\mathrm{d} g+c f}{f+g}\right) \lambda \\
& +2 a b(c-d)=0 .
\end{aligned}
$$

Denote three characteristic roots of (31) by $\lambda_{1}, \lambda_{2}$, and $\lambda_{3}$. Combining equations (27) and (31), we obtain

$$
\left\{\begin{array}{l}
\lambda_{1}+\lambda_{2}+\lambda_{3}=-(a+b-c), \\
\lambda_{1} \lambda_{2}+\lambda_{2} \lambda_{3}+\lambda_{1} \lambda_{3}=b\left(a-\frac{\mathrm{d} g+c f}{f+g}\right) \\
\lambda_{1} \lambda_{2} \lambda_{3}=2 a b(c-d) .
\end{array}\right.
$$

Then,

$$
H=\left[\begin{array}{ccc}
a+b-c & 2 a b(c-d) & 0 \\
1 & b\left(a-\frac{\mathrm{d} g+c f}{f+g}\right) & 0 \\
0 & a+b-c & 2 a b(c-d)
\end{array}\right],
$$

and the principal minors for $H$ are

$$
\left\{\begin{array}{l}
H_{1}=a+b-c \\
H_{2}=b\left[\left(a-\frac{\mathrm{d} g+c f}{f+g}\right)(a+b-c)-2 a(c-d)\right] \\
H_{3}=2 a b(c-d) H_{2} .
\end{array}\right.
$$

Let $H_{2}=(b p(c) /(f+g))$, where

$$
\begin{aligned}
p(c)= & f c^{2}-[3 a(g+f)-\mathrm{d} g+(a+b) f] c \\
& +(a+b)[a(g+f)-\mathrm{d} g]+2 a d(f+g) .
\end{aligned}
$$

For $p(c)$, we have the following properties:

(1) If $f=0$ and $d \leq a$, then $p(c)=0$ has a root $c_{0}$, which can be expressed as

$$
c_{0}=\frac{a^{2}+a(b+d)-b d}{3 a-d} .
$$

(2) If $f>0$, it follows that

(i) For $d<a, p(c)=0$ has two real roots $c_{ \pm}$, which can be expressed in this form:

$$
c_{ \pm}=\frac{3 a(g+f)-\mathrm{d} g+(a+b) f \pm \sqrt{[a(g+f)+(a+b) f+\mathrm{d} g]^{2}+8 a(a-d)(f+g)^{2}}}{2 f} .
$$

(ii) For $d=a$ and $2 a(g+f)+b f \neq 0, p(c)=0$ has

two real roots $c_{+}$, which can be expressed as

$$
c_{ \pm}=\frac{3 a(g+f)-\mathrm{d} g+(a+b) f \pm \sqrt{[a(g+f)+(a+b) f+\mathrm{d} g]^{2}+8 a(a-d)(f+g)^{2}}}{2 f} .
$$

(iii) For $d=a$ and $2 a(f+g)+b f=0, p(c)=0$ has a unique real root $\bar{c}=a$. Based on the above analysis, it is not difficult to deduce the following lemmas about $c_{0}$ and $c_{ \pm}$.
Lemma 6. When $b>0$ and $d<a$, it follows

(1) If $f=0$, then $d<c_{0}<a+b$.

(2) If $f>0$, then $d<c_{-}<a+b<c_{+}$. 
Lemma 7. When $b<0$ and $d<a$, then we have the following true statements:

(1) For $f=0$,

(i) If $a+b<d<a$, then $a+b<c_{0}<d$.

(ii) If $a+b=d<a$, then $a+b=c_{0}=d$.

(iii) If $d<a+b<a$, then $d<c_{0}<a+b$.

(2) For $f>0$,

(i) If $a+b<d<a$, then $a+b<c_{-}<d<c_{+}$.

(ii) If $a+b=d<a$, then $a+b=c_{-}=d<c_{+}$.

(iii) If $d<a+b<a$, then $d<c_{-}<a+b<c_{+}$.

Then, we can conclude the results in the following.

Theorem 4. Suppose $b>0$ and $d<a$, then we have the following true statements:

(1) For $f=0$,

(i) If $d<c<c_{0}$, then $E_{+}$is asymptotically stable.

(ii) If $c>c_{0}$, then $E_{+}$is unstable.

(iii) If $c=c_{0}$, then system (5) will undergo a Hopf bifurcation at $E_{+}$and a periodic orbit with period $T=\left(2 \pi / \sqrt{2 a b\left(c_{0}-d\right) /\left(a+b-c_{0}\right)}\right)$ will occur. The illustrative phase portraits are depicted in Figure 5.

(2) For $f>0$,

(i) If $d<c<c_{-}$, then $E_{+}$is asymptotical stable.

(ii) If $c>c_{-}$, then $E_{+}$is unstable.

(iii) If $c=c_{-}$, then system (5) undergoes a Hopf bifurcation at $E_{+}$and an orbit with periodic $T=$ $\left(2 \pi / \sqrt{2 a b\left(c_{-}-d\right) /\left(a+b-c_{-}\right)}\right)$will happen. The corresponding illustrative phase portraits are shown in Figure 6.

Proof. Based on Routh-Hurwitz criterion, we know the necessary and sufficient condition for $\lambda_{1}, \lambda_{2}$, and $\lambda_{3}$ to have negative real parts is $H_{1}, H_{2}, H_{3}>0$. When $b>0$, it follows from the existence of $E_{+}$that $c>d$. Then, due to (34), we achieve

$$
\left\{\begin{array}{l}
H_{1}=a+b-c \Leftrightarrow c<a+b, \\
H_{2}=b\left[\left(a-\frac{\mathrm{d} g+c f}{f+g}\right)(a+b-c)-2 a(c-d)\right] \Leftrightarrow p(c)>0, \\
H_{3}=2 a b(c-d) H_{2} \Leftrightarrow H_{2}>0 .
\end{array}\right.
$$

For $f=0$, based on Lemma 6 (1), we know that $d<c_{0}<a+b$ holds. Hence, for $d<c<c_{0}$, we have $H_{1}, H_{2}$, $H_{3}>0$, which shows $E_{+}$is asymptotical stable, while, for $c>c_{0}$, it is easy to derive $H_{2}<0$; therefore, Lemma 5 (3) implies that has at least a root $\lambda_{i}$ with $\operatorname{Re}\left(\lambda_{i}\right)>0(i \in\{1,2,3\})$, so $E_{+}$is unstable. Thus, we complete the proofs of (1) (i) and (1) (ii).
For $f>0$, the proofs of (2) (i) and (2) (ii) are similar to that of (1) (i) and (1) (ii), so we omitted them here.

Next, we prove (2) (iii). For $f>0$ and $c=c_{-}$, we know that $k(\lambda)$ has a pair of pure imaginary roots $\lambda_{1,2}= \pm \sqrt{2 a b\left(c_{-}-d\right) /\left(a+b-c_{-}\right)} i= \pm \sqrt{\omega} i$ and one negative real root $\lambda_{3}=c_{-}-(a+b)$. Complicated calculations yield

$$
\left.\frac{\mathrm{d} \operatorname{Re}\left(\lambda_{1}\right)}{\mathrm{d} c}\right|_{c=c_{-}}=\frac{b f\left(a+b-c_{-}\right)+(f+g)(\omega+2 a b)}{2(f+g)\left[\omega+\left(a+b-c_{-}\right)^{2}\right]}>0 .
$$

According to the Hopf bifurcation theory [31], one knows that a limit cycle occurs at $c=c_{-}$, which increases with respect to $c$ and has the initial period $T=\left(2 \pi / \sqrt{2 a b\left(c_{-}-d\right) /\left(a+b-c_{-}\right)}\right)$. The above arguments imply that a Hopf bifurcation will happen at $E_{+}$of system (5). Using the same method as (2) (iii), we can prove the results of (1) (iii).

Theorem 5. Suppose $b<0$ and $d<a$, then $E_{+}$is unstable. The corresponding illustrative phase portraits are depicted in Figure 7.

Proof. When $b<0$, in order to guarantee the existence of $E_{+}$, we require $c<d$. If $f=0$, based on the statements of Lemma $7(1)$, we know that there exists at least a $H_{i}<0(i=1,2,3)$; then, according to Lemma 5 (3), we get that $k(\lambda)=0$ has at least one root with positive real part; thus, $E_{+}$is unstable. If $f>0$, with similar argument to the proof of $f=0$, combining Lemma 7 (2) and Lemma 5 (3), we achieve the unstability of $E_{+}$. Consequently, we finish the proof of Theorem 5.

Theorem 6. Assume $d=a$, then $E_{+}$is unstable. The corresponding illustrative phase portraits are depicted in Figure 8.

Proof. If $d=a$, let us consider $b>0$ and $b<0$, respectively, as follows:

Case $1(b>0)$ : it follows from the existence of $E_{+}$that $c>a$ holds. If $f=0$, then $c_{0}=a$, which means $c>c_{0}=a$; thus, $H_{2}<0$. If $f>0$, then $p(c)=0$ has two real roots, which leads to $a=c_{-}<a+b<c_{+}$; thus, when $a<c \leq a+b$, we have $H_{2}<0$, while, for $c>a+b$, we may obtain $H_{1}<0$. Hence, according to the results of Lemma 5 (3), we know that $k(\lambda)$ has at least one root $\lambda_{i}(i \in\{1,2,3\})$ with $\operatorname{Re}\left(\lambda_{i}\right)>0$. So, $E_{+}$is unstable.

Case $2(b<0)$ : the condition $c<a$ is provided by the existence of $E_{+}$. If $f=0$, then $c_{0}=a$, which leads to $c<c_{0}=a$; thus, $H_{2}<0$. If $f>0$ and $2 a(f+g)+b f=0$, then $p(c)=0$ has one real root $\bar{c}$ and $c<\bar{c}=a$, which shows $H_{2}<0$. If $f>0$ and $2 a(f+g)+b f>0$, then $p(c)=0$ has two real roots and $a+b<c_{-}$, so, when $c \geq c_{-}$, we have $H_{1}<0$, while, for $c<c_{-}, H_{2}<0$ is fulfilled. If $f>0$ and $2 a(f+g)+b f<0$, then $p(c)=0$ has two real roots and $a+b<c_{+}$; hence, for $c \geq c_{+}, H_{1}<0$ holds, while, for $c<c_{+}, H_{2}<0$ is true. Therefore, based on the 


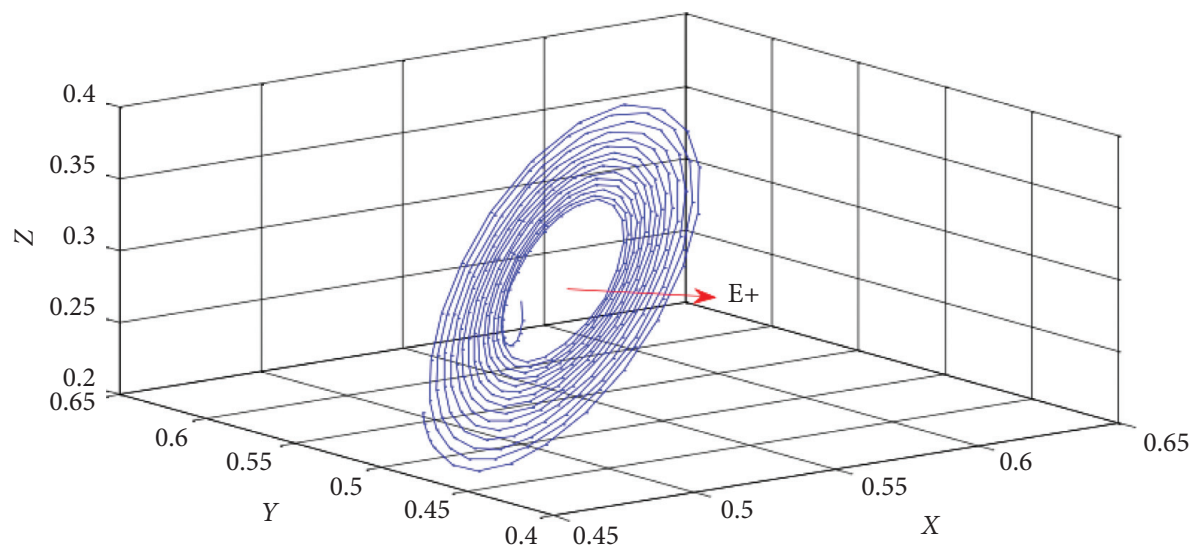

(a)

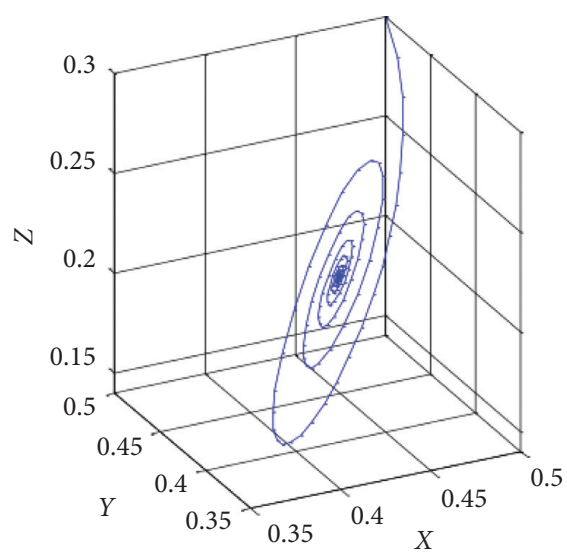

(b)

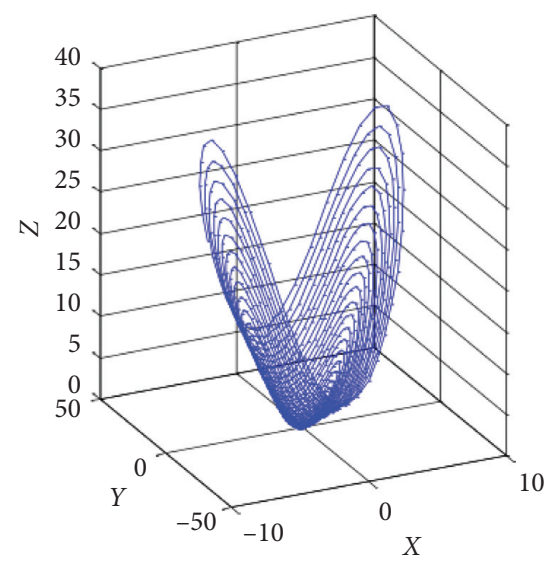

(c)

Figure 5: Phase portraits for system (5) when $(a, b, d, f, g)=(0.8,1,0.4,0,1)$ and (a) $c=c_{0}=0.69$, (b) $c=0.6<c_{0}$, and (c) $c=0.8>c_{0}$.

conclusion of Lemma 5 (3), we get that $k(\lambda)=0$ has at least one root $\lambda_{i}(i \in\{1,2,3\})$ with $\operatorname{Re}\left(\lambda_{i}\right)>0$. Hence, the equilibrium point $E_{+}$is unstable, which finishes Theorem 6.

Because $E_{+}$and $E_{-}$are symmetrical, the following results can be achieved.

Theorem 7. When $a>0, b, c, d \in \mathbb{R}, d \leq a, g \geq 0, f \geq 0$, and $g+f>0$, the local dynamical properties of the equilibrium points $E_{ \pm}$are summed up in Table 4.

Remark 2. For the case $d>a$, we cannot find an effective method to classify the parameters to investigate the dynamical behavior of $E_{ \pm}$, but we present numerical simulation for parameters $(a, b, c, d, f, g)=(2,1,4,3,0,1)$ and $(a, b, c$, $d, f, g)=(2,-1,3,4,0,1)$ which are shown in Figure 9.

\section{Dynamics at Infinity on System (5)}

In this section, the dynamics at infinity on system (5) will be discussed with the aid of the way to Poincaré compactification in $\mathbb{R}^{3}[32,33]$.
3.1. The Infinite Equilibrium Points of System (5). To study the dynamics at infinity, we should investigate the Poincaré compactification for system (5) in the local charts $U_{i}$ and $V_{i}(i=1,2,3)$, respectively.

3.1.1. In the Local Charts $U_{1}$ and $V_{1}$. Based on $[32,33]$, the Poincaré compactification $p(X)$ for system (5) in the chart $U_{1}$ may be displayed as

$$
\left\{\begin{array}{l}
\dot{z}_{1}=-z_{2}-\mathrm{d} z_{3}+(a+c) z_{1} z_{3}-a z_{1}^{2} z_{3}, \\
\dot{z}_{2}=f+g z_{1}+(a-b) z_{2} z_{3}-a z_{1} z_{2} z_{3}, \\
\dot{z}_{3}=a z_{3}^{2}-a z_{1} z_{3}^{2} .
\end{array}\right.
$$

As we know, to study the points of the sphere $S^{2}$ at infinity, one needs to set $z_{3}=0$; it then follows that

$$
\left\{\begin{array}{l}
\dot{z}_{1}=-z_{2}, \\
\dot{z}_{2}=f+g z_{1} .
\end{array}\right.
$$

One may get that system (42) has no equilibrium point for $g=0$, while, for $g>0$, system (42) has a unique equilibrium point $(-(f / g), 0)$. That is to say, system $(41)$ has no equilibrium point for $g=0$ and has a single equilibrium point $P_{1}(-(f / g), 0,0)$ for $g>0$. Moreover, a first integral, 


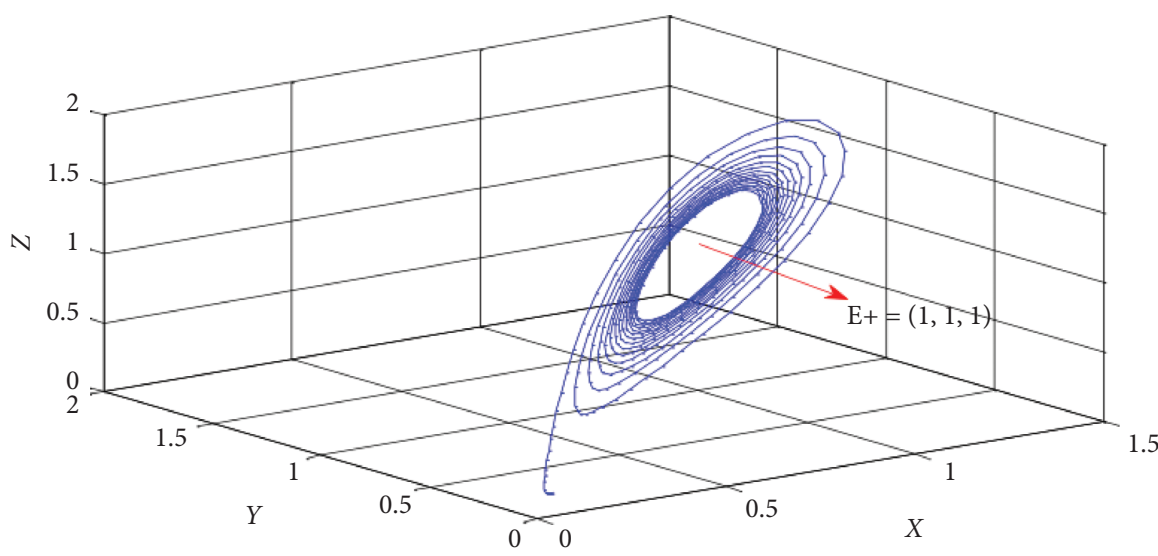

(a)

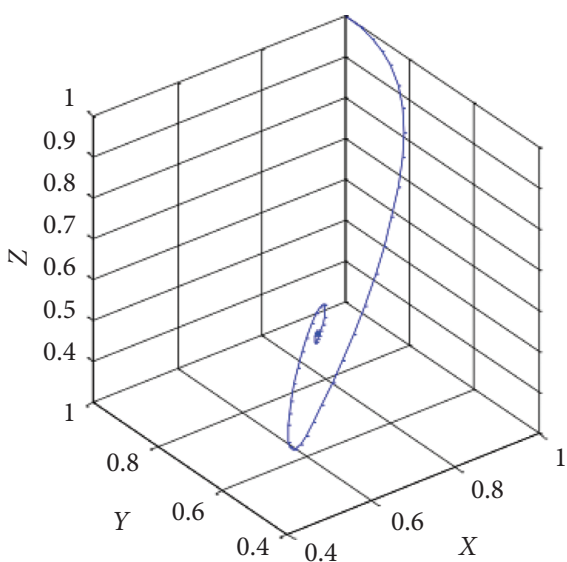

(b)

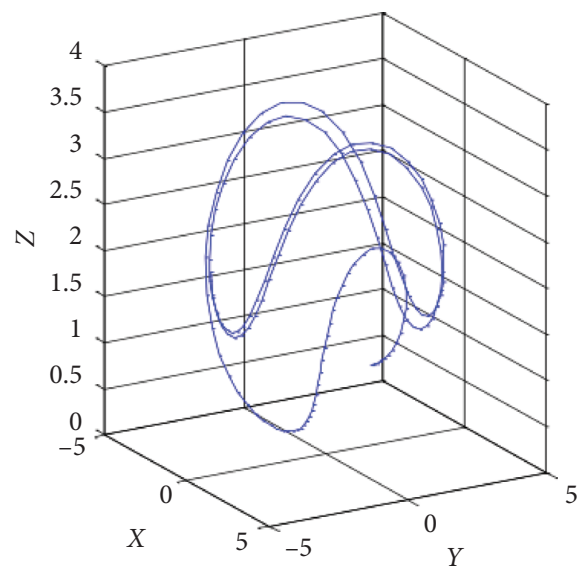

(c)

Figure 6: Phase portraits for system (5) when $(a, b, d, f, g)=(1,1,-1,1,0)$ and (a) $c=c_{-}=0$, (b) $c=-0.5<c_{-}$, and (c) $c=0.5>c_{-}$.

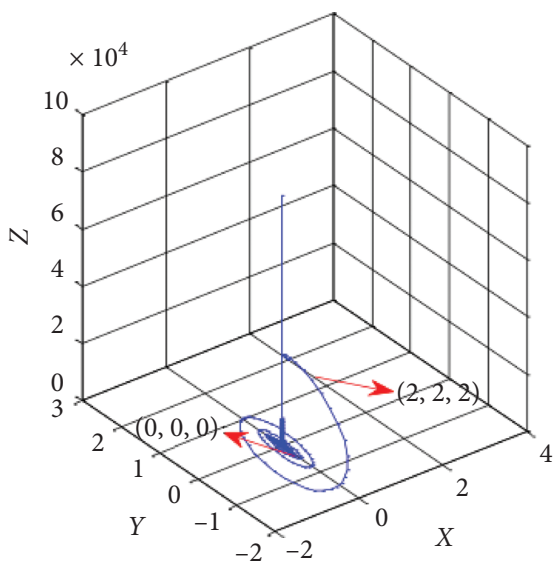

(a)

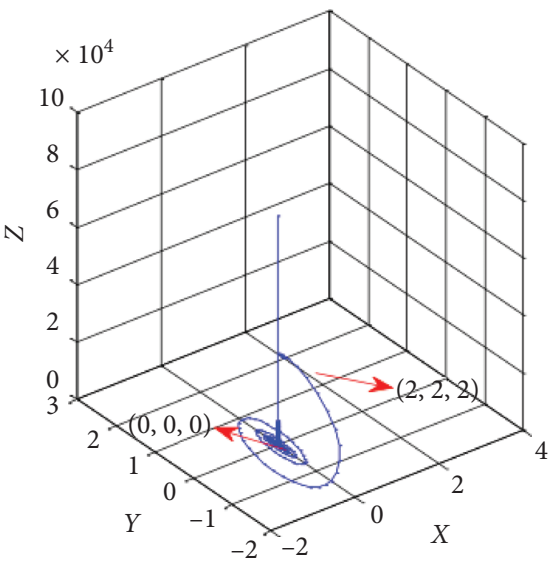

(b)

Figure 7: Phase portraits for system (5) when $a>d$ and (a) $(a, b, c, d, f, g)=(3,-1,1,2,1,0)$ and $(\mathrm{b})(a, b, c, d, f, g)=(3,-1,1,2,0,1)$.

$$
H\left(z_{1}, z_{2}\right)=\left(z_{1}+\frac{f}{g}\right)^{2}+\frac{z_{2}^{2}}{g}
$$

has been found in system (42), which shows the equilibrium point $P_{1}$ of system (41) is a center. And, the phase portrait for (43) has been drawn in Figure 10, which helps us to get the corresponding phase portrait for our system (5) on the sphere at infinity.

In order to find out the dynamics of the local property of $P_{1}$, we can analyze the flow on the center manifold related to its eigenvalue 0 and achieve the statement listed as follows. 


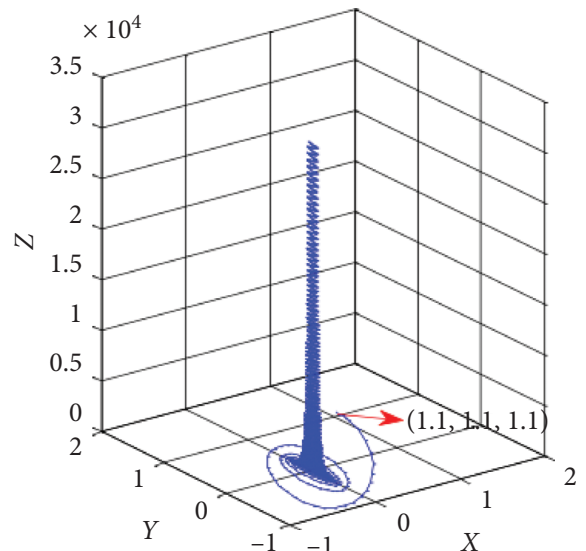

(a)

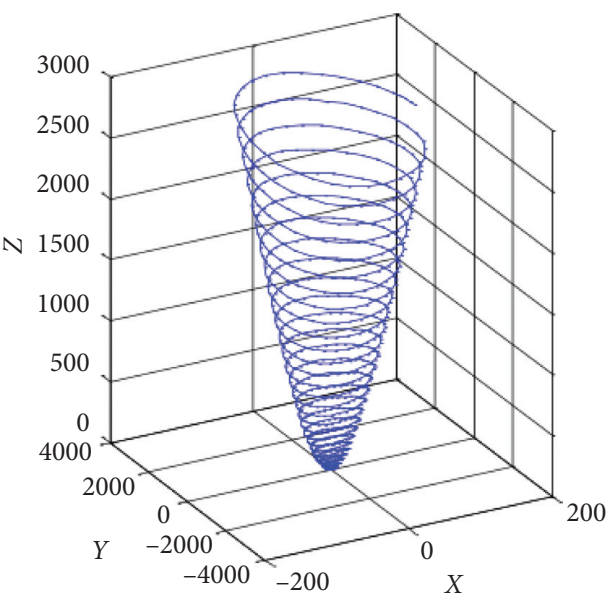

(c)

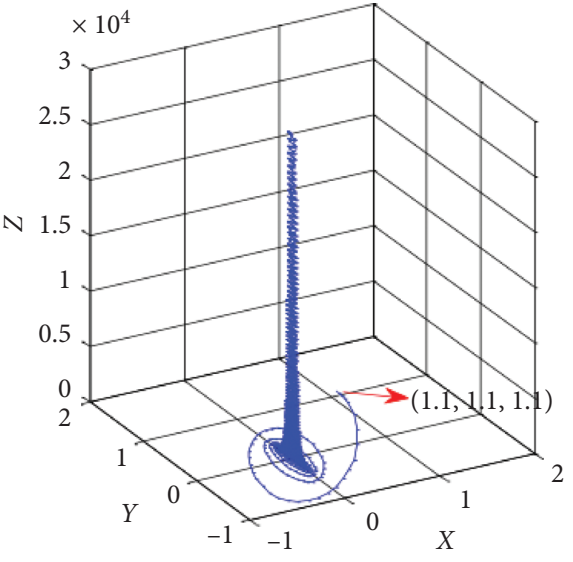

(b)

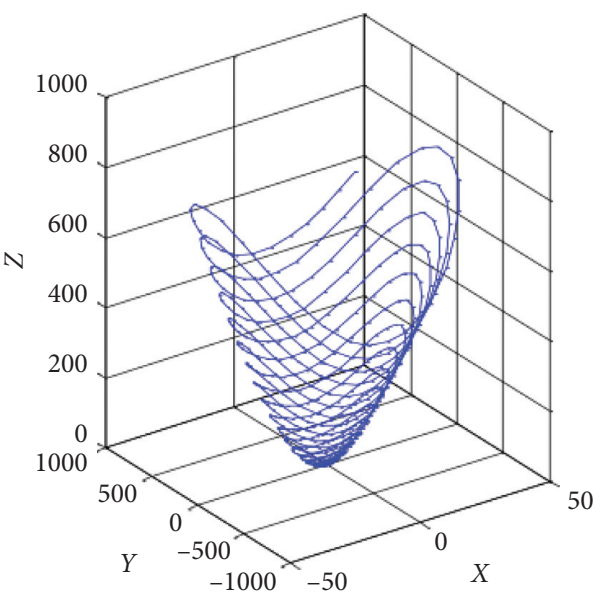

(d)

Figure 8: Phase portraits for system (5) when $a=d$ and (a) $(a, b, c, d, f, g)=(3,-1,2,3,1,0),(\mathrm{b})(a, b, c, d, f, g)=(3,-1,2,3,0,1),(\mathrm{c})$ $(a, b, c, d, f, g)=(3,1,4,3,1,0)$, and (d) $(a, b, c, d, f, g)=(3,1,4,3,0,1)$.

TABLE 4: Local dynamical properties of $E_{+}$.

\begin{tabular}{ccccc}
\hline$b$ & $d-a$ & $f$ & $c$ & Dynamical behavior of $E_{ \pm}$ \\
\hline & & & $c<c_{0}$ & Asymptotical stable \\
& & & $c=c_{0}$ & Hopf bifurcation \\
& & & $c>c_{0}$ & Unstable \\
$>0$ & $<0$ & & $c<c_{-}$ & Asymptotical stable \\
& & $>0$ & $c=c_{-}$ & Hopf bifurcation \\
& & & $c>c_{-}$ & Unstable \\
& & & & Unstable \\
\hline$<0$ & $\leq 0$ & & & Unstable \\
\hline
\end{tabular}

Lemma 8. When $g>0$, the equilibrium point $P_{1}$ of system (40) has a $1 D$ center manifold $W_{\text {loc }}^{c}$, and it is unstable along $W_{l o c}^{c}$.

Proof. Since the equilibrium point $P_{1}$ has eigenvalues $\lambda_{1,2}=$ $\pm \sqrt{g} i$ and $\lambda_{3}=0$, which implies $P_{1}$ has a $1 \mathrm{D} W_{\text {loc }}^{c}$, set $k_{1}=z_{1}+(f / g), k_{2}=z_{2}$, and $k_{3}=z_{3}$, which translates the singular point $P_{1}$ into the origin $O(0,0,0)$. Then, system (41) is converted into

$$
\left\{\begin{array}{l}
\dot{k}_{1}=-k_{2}-\mathrm{d} k_{3}+(a+c)\left(k_{1}-(f / g)\right) k_{3}-a\left(k_{1}-(f / g)\right)^{2} k_{3}, \\
\dot{k}_{2}=g k_{1}+(a-b) k_{2} k_{3}-a\left(k_{1}-(f / g)\right) k_{2} k_{3}, \\
\dot{k}_{3}=a k_{3}^{2}-a\left(k_{1}-(f / g)\right) k_{3}^{2} .
\end{array}\right.
$$

Thus, the stability of $P_{1}$ restricted on the one-dimensional center manifold can be established according to that of the origin on a center manifold. Assume that the graph of a function $\quad h: \mathbb{R} \longrightarrow \mathbb{R}^{2} \quad$ is presented with $\left(k_{1}, k_{2}\right)=h\left(k_{3}\right)=\left(h_{1}\left(k_{3}\right), h_{2}\left(k_{3}\right)\right)$, fulfilling

$$
\begin{aligned}
h(0) & =(0,0), \\
D h(0) & =(0,-m) .
\end{aligned}
$$

Expand $h$ at $k_{3}=0$ with Taylor series, which gives

$$
\begin{aligned}
& h_{1}\left(k_{3}\right)=\sum_{i=2}^{\infty} a_{i} k_{3}^{i}, \\
& h_{2}\left(k_{3}\right)=-m k_{3}+\sum_{i=2}^{\infty} a_{i} k_{3}^{i} .
\end{aligned}
$$




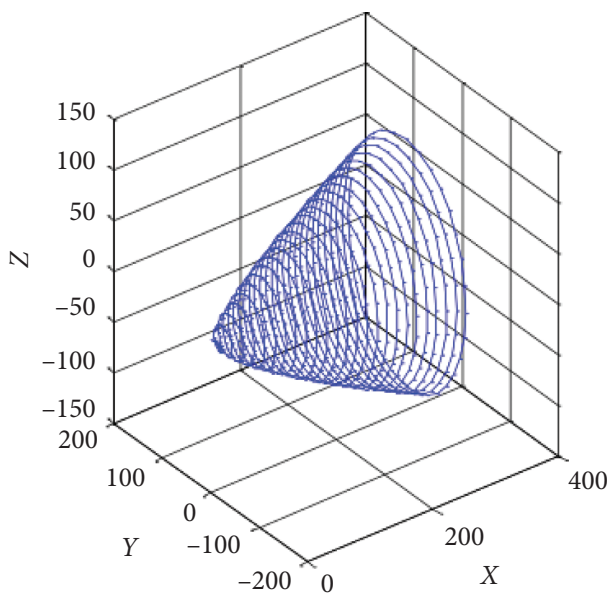

(a)

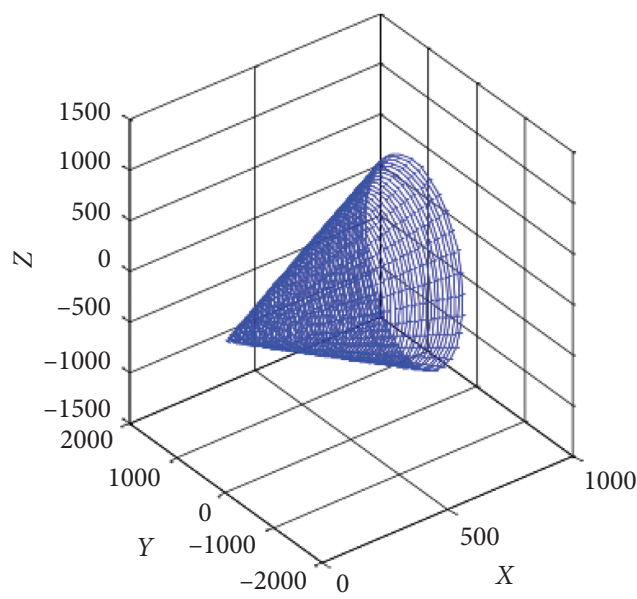

(b)

Figure 9: Phase portraits for system (5) when the parameters satisfy (a) $(a, b, c, d, f, g)=(2,1,4,3,0,1)$ and $(\mathrm{b})(a, b, c, d, f, g)=$ $(2,-1,3,4,0,1)$.

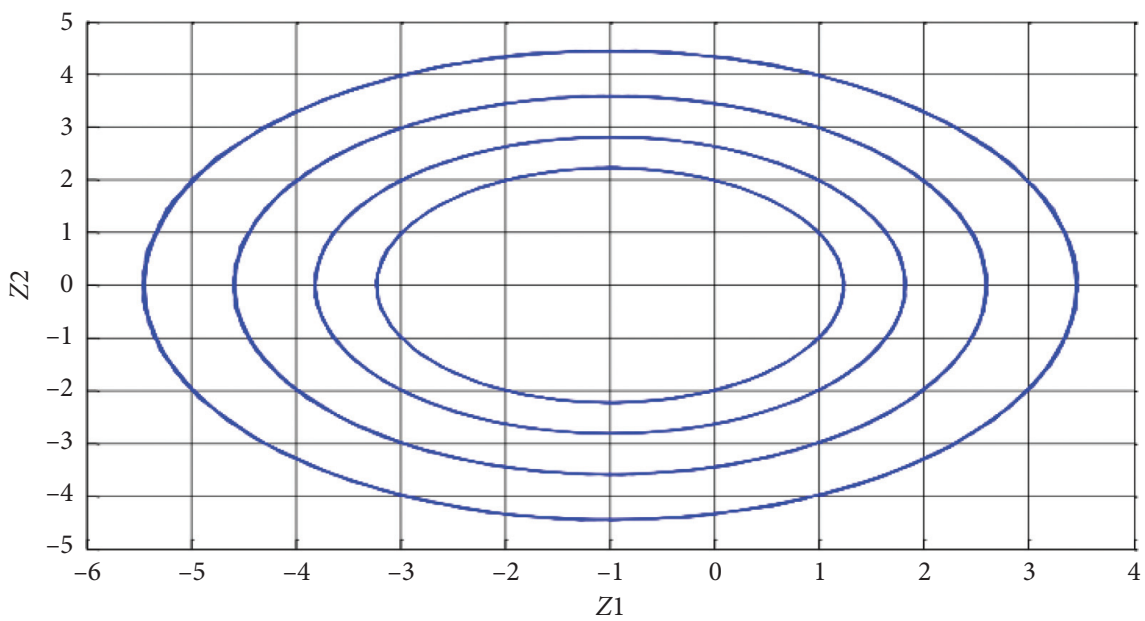

Figure 10: The phase portrait of $H\left(z_{1}, z_{2}\right)=\left(z_{1}+(f / g)\right)^{2}+\left(z_{2}^{2} / g\right)$ when $(f, g)=(1,1)$.

Substituting them into (44) and comparing the coefficients of $k_{3}$ on both sides, we obtain

$$
\left\{\begin{array}{l}
h_{1}\left(k_{3}\right)=-\frac{m b}{g} k_{3}^{2}+O\left(k_{3}^{4}\right), \\
h_{2}\left(k_{3}\right)=\frac{(a-c) m b}{g} k_{3}^{3}+O\left(k_{3}^{4}\right) .
\end{array}\right.
$$

Then, we derive $\dot{k}_{3}=a(1+(f / g)) k_{3}^{2}+O\left(k_{3}^{4}\right)$, which implies that when $z_{3}>0, P_{1}$ is local unstable along its center manifold.

Notice that multiplying -1 in the compactified vector field $p(X)$ in $U_{1}$ can give the compactified vector field $p(X)$ in $V_{1}$, so the flow in $V_{1}$ is the same as the flow in $U_{1}$ if we reverse the time. We have to point out that $z_{3}<0$ should be considered in the neighborhood of infinity in $V_{1}$, see Figure 11.
3.1.2. In the Local Charts $U_{2}$ and $V_{2}$. The Poincaré compactification $p(X)$ for system (5) in $U_{2}$ has the following form:

$$
\left\{\begin{array}{l}
\dot{z}_{1}=a z_{3}-(a+c) z_{1} z_{3}+\mathrm{d} z_{1}^{2} z_{3}+z_{1}^{2} z_{2}^{2}, \\
\dot{z}_{2}=-(b+c) z_{2} z_{3}+\mathrm{d} z_{1} z_{2} z_{3}+g z_{1}+f z_{1}^{2}+z_{1} z_{2}^{2}, \\
\dot{z}_{3}=-c z_{3}^{2}+z_{1} z_{2} z_{3}+\mathrm{d} z_{1} z_{3}^{2} .
\end{array}\right.
$$

By setting $z_{3}=0$, system (48) becomes

$$
\left\{\begin{array}{l}
\dot{z}_{1}=z_{1}^{2} z_{2}, \\
\dot{z}_{2}=g z_{1}+f z_{1}^{2}+z_{1} z_{2}^{2} .
\end{array}\right.
$$

By some simple computations, one knows that, for $g=0$ and $f>0$ or $g>0$ and $f=0$, system (49) has a line of equilibrium points $\left(0, z_{2}\right)$, while, for $g>0$ and $f>0$, system (48) has an equilibrium point $(-(g / f), 0)$ except the line of equilibrium points $\left(0, z_{2}\right)$, which means system $(48)$ has a 


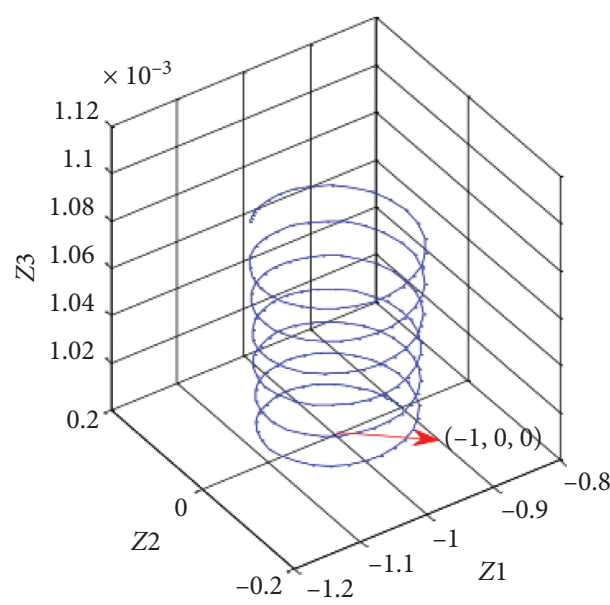

(a)

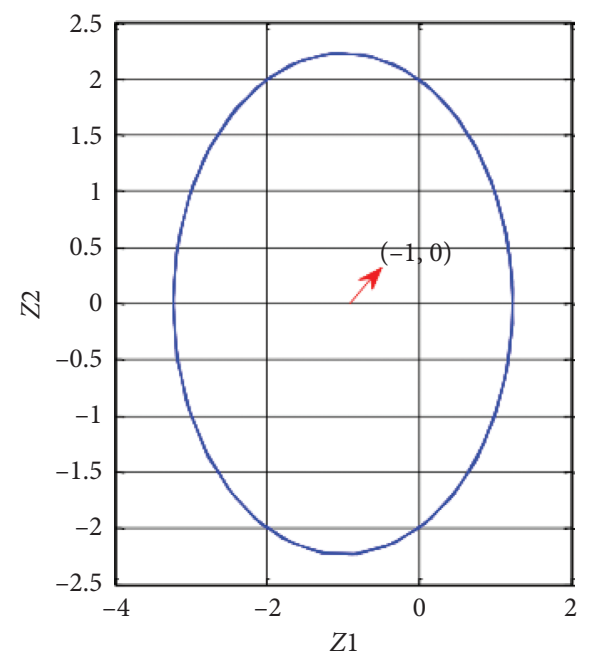

(b)

Figure 11: (a) Phase portrait for system $(40)$ when $(a, b, c, d, f, g)=(1,1,1,1,1,1)$. (b) Phase portrait of system $(42)$ with $(f, g)=(1,1)$.

line of equilibria $Q_{1}\left(0, z_{2}, 0\right)$ for $g=0$ and $f>0$ or $g>0$ and $f=0$, while, for $g>0$ and $f>0$, system (48) has a line of the equilibrium points $Q_{1}\left(0, z_{2}, 0\right)$ and an isolated equilibrium point $Q_{2}(-(g / f), 0,0)$.

Moreover, system (49) has a first integral,

$$
H\left(z_{1}, z_{2}\right)=\frac{z_{2}^{2}+2 f z_{1}+g}{z_{1}^{2}},
$$

which helps us to get the phase portrait of system (51), one may see Figure 12.

Similar to the situation of $U_{1}$ and $V_{1}$, we can get the flow of system (5) in $V_{2}$.

3.1.3. In the Local Charts $U_{3}$ and $V_{3}$. Similarly, one may has the Poincare compactification $p(X)$ for system (5) in the local chart $U_{3}$ listed as

$$
\left\{\begin{array}{l}
\dot{z}_{1}=(b-a) z_{1} z_{3}+a z_{2} z_{3}-f z_{1}^{3}-g z_{1}^{2} z_{2}, \\
\dot{z}_{2}=-\mathrm{d} z_{1} z_{3}+(b+c) z_{2} z_{3}-z_{1}-f z_{1}^{2} z_{2}-g z_{1} z_{2}^{2}, \\
\dot{z}_{3}=b z_{3}^{2}-g z_{1} z_{2} z_{3}-f z_{1}^{2} z_{3},
\end{array}\right.
$$

which turns into the following form with $z_{3}=0$ :

$$
\left\{\begin{array}{l}
\dot{z}_{1}=-f z_{1}^{3}-g z_{1}^{2} z_{2}, \\
\dot{z}_{2}=-z_{1}-f z_{1}^{2} z_{2}-g z_{1} z_{2}^{2} .
\end{array}\right.
$$

It is not difficult to see that there is a first integral in system (52):

$$
H\left(z_{1}, z_{2}\right)=\frac{g z_{2}^{2}+2 f z_{1} z_{2}}{z_{1}^{2}},
$$

and then, the phase portrait of system (52) will be achieved, which is shown in Figure 13.

With similar arguments above, we know that system (51) has a line of the equilibrium points $R_{1}\left(0, z_{2}, 0\right)$. Moreover, the dynamics in the local charts $V_{3}$ may be easily obtained.
Summarizing the analysis provided above, one may achieve the following result about the infinite dynamics of system (5) on the sphere.

Theorem 8. Suppose $g \geq 0, f \geq 0$, and $g+f>0$, the infinite dynamical behavior of system (5) on the sphere is summarized in Figure 14.

3.2. The Existence of Infinite Heteroclinic Orbits. Now, we study how the orbits in the vicinity of the Poincaré ball reach to the equilibrium point of the sphere along $z$-axis. In fact, we only need to study how the orbits in a neighborhood of $\left(0,0, z_{3}\right)\left(0<z_{3} \ll 1\right)$ in system $(26)$ tend to the origin on the chart $U_{3}$.

From the corresponding relation between the local chart $U_{3}$ and the hemispherical surface, we have

$$
\left\{\begin{array}{l}
y_{1}=z_{1} \Delta z, \\
y_{2}=z_{2} \Delta z, \\
y_{3}=\Delta z, \\
y_{4}=z_{3} \Delta z,
\end{array}\right.
$$

where $y_{4}>0$ and $\Delta z=\left(1+z_{1}^{2}+z_{2}^{2}+z_{3}^{2}\right)^{-(1 / 2)}$. Let $z_{1}=0$, $z_{2}=0$, and $0<z_{3} \ll 1$; then, $y_{1}=y_{2}=0, y_{3} \longrightarrow 1$, and $y_{4} \longrightarrow 0$ as $z_{3} \longrightarrow 0$. This means that the axial lines in the interior of the Poincare sphere tend to the endpoint of the positive $z$-axis. Hence, we get the following results about the infinite equilibria.

Lemma 9. The equilibrium points at the endpoints of $z$-axis on the sphere at infinity are asymptotically stable (resp. unstable) along $z$-axis for $b<0$ (resp. $b>0)$.

Proof. Since the equilibrium point is at the end point of positive (resp. negative) $z$-axis on the sphere at infinity coincide with the origin of the Poincaré compactification $p(X)$ for system (5) in the local chart $U_{3}$ (resp. $V_{3}$ ), we deal 


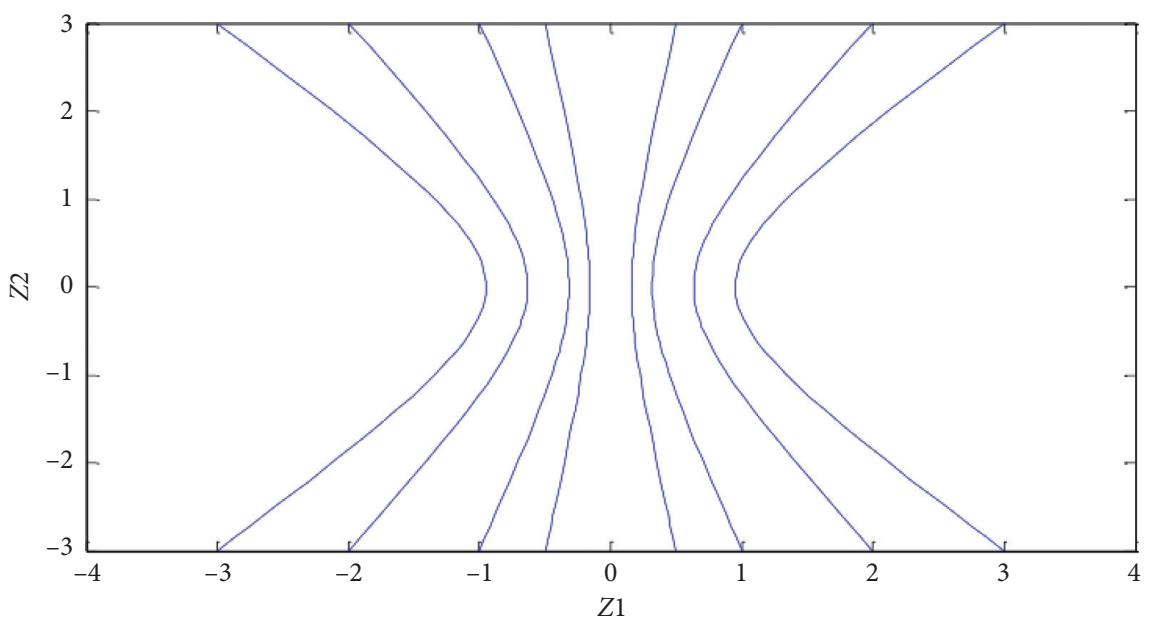

Figure 12: The phase portrait for system (49) when $(f, g)=(0,1)$.

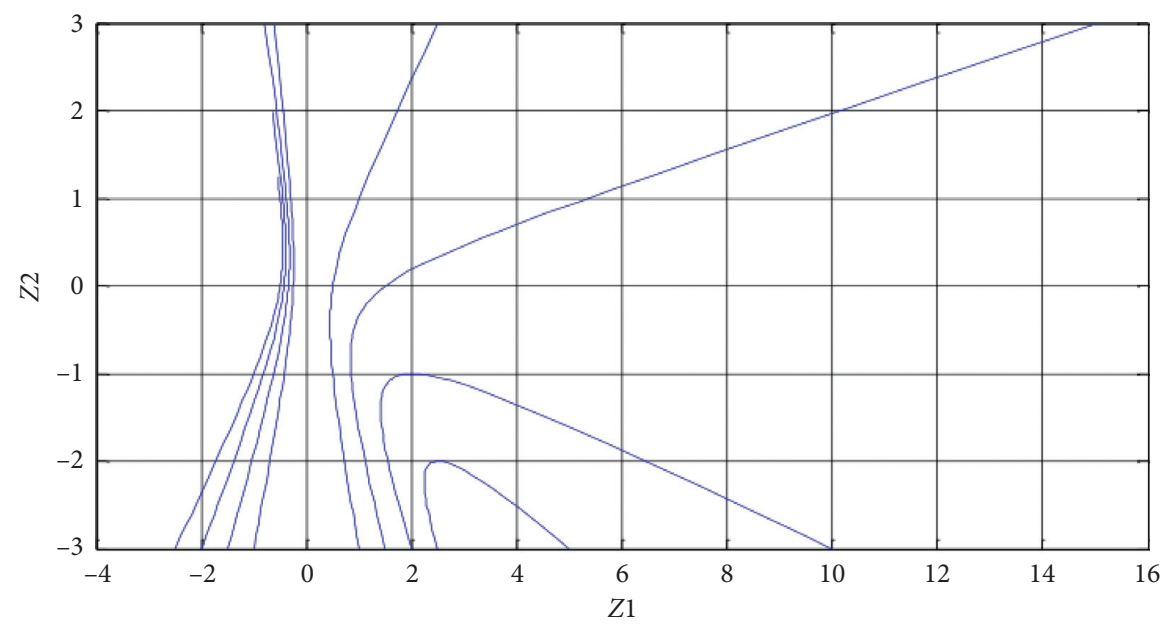

Figure 13: The phase portrait for system (27) when $(f, g)=(1,1)$.

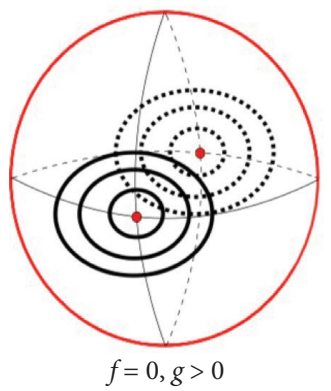

(a)

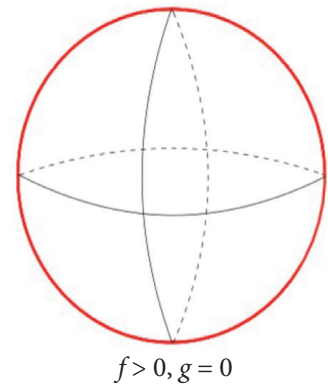

(b)

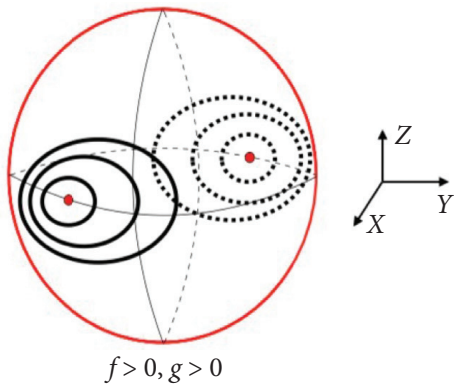

(c)

Figure 14: Phase portraits for system (5) at infinity. (a) $f=0, g>0$, (b) $f>0, g=0$, and (c) $f>0, g>0$. 


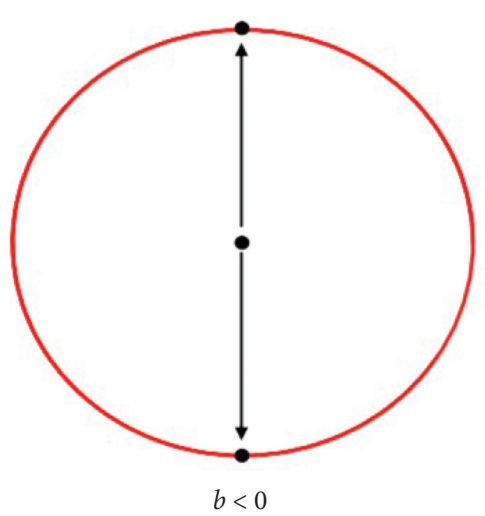

(a)

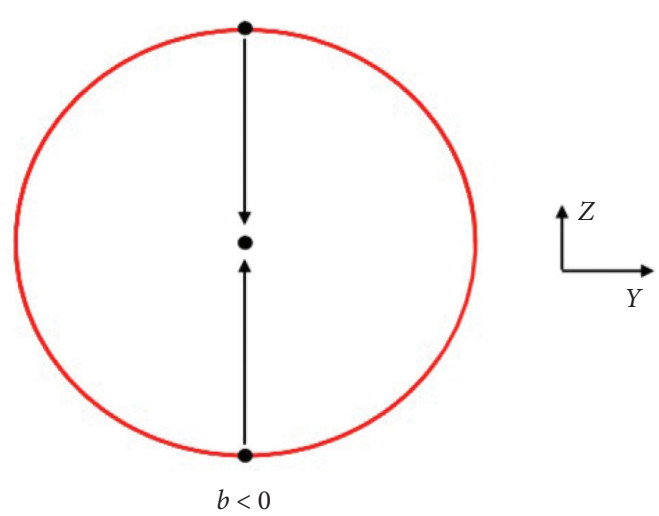

(b)

FIgURe 15: Infinite heteroclinic orbits for system (5) when $b<0$ and $b>0$.

with the origin $I_{0}(0,0,0)$ for system (51) first. Obviously, $z_{3}$-axis is invariant under the flow of (51); notice, for $z_{1}=0$ and $z_{2}=0,(51)$ is reduced to

$$
\left\{\begin{array}{l}
\dot{z}_{1}=0, \\
\dot{z}_{2}=0, \\
\dot{z}_{3}=b z_{3}^{2} .
\end{array}\right.
$$

Hence, $I_{0}(0,0,0)$ is asymptotically stable for $b<0$, which means that orbits tend to $I_{0}$ along $z_{3}$-axis from $z_{3}>0 ; I_{0}=$ $(0,0,0)$ is unstable for $b>0$, which means that orbits are far from $I_{0}$ along $z_{3}$-axis from $z_{3}>0$.

As to the equilibrium point at the endpoint of negative $z$-axis, we should consider

$$
\left\{\begin{array}{l}
\dot{z}_{1}=0, \\
\dot{z}_{2}=0, \\
\dot{z}_{3}=-b z_{3}^{2},
\end{array}\right.
$$

where we need to take into account the case $z_{3}<0$. The proof can be finished then.

Based on the conclusions obtained above, we may achieve the following result about infinite heteroclinic orbits.

Theorem 9. If $b \neq 0$, then there exist two infinite heteroclinic orbits denoted by $\Gamma_{i}(i=1,2)$ for system (5): $\Gamma_{1}$ connects the origin $E_{0}$ and the endpoint of the positive $z$-axis along the positive part of $z$-axis; $\Gamma_{2}$ connects the origin $E_{0}$ and the endpoint of the negative $z$-axis along the negative part of $z$-axis. Furthermore, the origin is unstable for $b<0$ and stable for $b>0$, one may see Figure 15.

Proof. When $b \neq 0, c, d \in \mathbb{R}$, we have presented the dynamical properties of the origin $E_{0}$ in Table 2. The eigenvalues of Jacobian matrix for system (5) at $E_{0}$ are

$$
\begin{aligned}
& \lambda_{1,2}=\frac{c-a \pm \sqrt{(a-c)^{2}+4 a(c-d)}}{2}, \\
& \lambda_{3}=-b,
\end{aligned}
$$

and the corresponding eigenvectors can be taken as

$$
\begin{aligned}
& v_{1,2}=\left(\frac{-a-c \pm \sqrt{(a-c)^{2}+4 a(c-d)}}{-2 d}, 1,0\right), \\
& v_{3}=(0,0,1) .
\end{aligned}
$$

Next, we only give the proof of the situation $b<0$ here; for $b>0$, we may finish the proof similarly.

Notice that the eigenvalue $\lambda_{3}=-b>0$ for $b<0$, which means that the instability index of $E_{0}$ is at least 1 . It is not difficult to see from system (5) that $z$-axis is invariant, which directly leads to the instability along $z$-axis of the equilibrium point $E_{0}$. Furthermore, we have proved that the equilibrium points at the endpoints of $z$-axis on the sphere at infinity are asymptotically stable. Consequently, there exist two infinite heteroclinic orbits for system (5), and we may denote them by $\Gamma_{1}$ and $\Gamma_{2}$, respectively. Moreover, $\Gamma_{1}$ connects the origin and the endpoint of the positive $z$-axis along positive part of the $z$-axis, while $\Gamma_{2}$ connects the origin and the endpoint of the negative $z$-axis along negative part of the $z$-axis. These are illustrated in Figure 15.

\section{Conclusion and the Future Work}

The paper proposed a new three-dimensional Lorenz-like chaotic system with eight terms; some interesting and important dynamics of this system were dug out. By combining theoretical analysis and numerical simulations, we rigorously investigated the dynamics at finity and at infinity of system (5). More exactly, the classification of the equilibrium points, the local properties of the nonhyperbolic equilibrium points, the Hopf bifurcations, and the expressions of the local manifolds were clearly provided. As to the dynamics at infinity, we analyzed the flow of the proposed system near and at infinity. Particularly, the existence of infinite heteroclinic orbits was studied by combining the dynamic at finity and infinity.

Even so, there are still more important research studies to be carried on in the further work, such as the theoretical 
proof to the existence of the chaos, chaos control, and applications of our studied system.

\section{Data Availability}

No data were used to support the study.

\section{Conflicts of Interest}

The authors declare that they have no conflicts of interest.

\section{Acknowledgments}

This research was supported by the National Natural Science Foundation of China (nos. 12001503 and 12001117), Fundamental Research Funds for the Central Universities (no. 2652018055), and Project of Beijing Municipal Commission of Education (no. KM202110015001).

\section{References}

[1] G. Álvarez, S. Li, F. Montoya, G. Pastor, and M. Romera, "Breaking projective chaos synchronization secure communication using filtering and generalized synchronization," Chaos, Solitons \& Fractals, vol. 24, no. 3, pp. 775-783, 2005.

[2] R. C. Eberhart, "Chaos theory for the biomedical engineer," IEEE Engineering in Medicine and Biology Magazine, vol. 3, pp. 41-45, 2002.

[3] L. M. Pecora and T. L. Carroll, "Synchronization in chaotic systems," Physical Review Letters, vol. 64, no. 8, pp. 821-824, 1990.

[4] J. M. Ottino, C. W. Leong, H. Rising, and P. D. Swanson, "Morphological structures produced by mixing in chaotic flows," Nature, vol. 333, no. 6172, pp. 419-425, 1988.

[5] O. Mofid, S. Mobayen, and M. H. Khooban, "Sliding mode disturbance observer control based on adaptive synchronization in a class of fractional-order chaotic systems," International Journal of Adaptive Control and Signal Processing, vol. 33, no. 3, pp. 462-474, 2019.

[6] S. Mobayen, S. Vaidyanathan, A. Sambas, S. Kaçar, and Ü. Çavuşoğlu, "A novel chaotic system with boomerang-shaped equilibrium, its circuit implementation and application to sound encryption," Iranian Journal of Science and Technology, Transactions of Electrical Engineering, vol. 43, no. 1, pp. 1-12, 2019.

[7] B. Vaseghi, M. A. Pourmina, and S. Mobayen, "Finite-time chaos synchronization and its application in wireless sensor networks," Transactions of the Institute of Measurement and Control, vol. 40, no. 3, pp. 1-12, 2017.

[8] S. Mobayena and J. Mab, "Robust finite-time composite nonlinear feedback control for synchronization of uncertain chaotic systems with nonlinearity and time-delay," Chaos, Solitons and Fractals, vol. 114, pp. 46-54, 2018.

[9] J. Lü and G. Chen, "A new chaotic attractor coined," International Journal of Bifurcation and Chaos, vol. 12, no. 3, pp. 659-661, 2002.

[10] G. Chen and T. Ueta, "Yet another chaotic attractor," International Journal of Bifurcation and Chaos, vol. 9, no. 7, pp. 1465-1466, 1999.

[11] X. Li and Q. Ou, "Dynamical properties and simulation of a new Lorenz-like chaotic system," Nonlinear Dynamics, vol. 65, no. 3, pp. 255-270, 2011.
[12] Y. Liu and Q. Yang, "Dynamics of a new Lorenz-like chaotic system," Nonlinear Analysis: Real World Applications, vol. 11, no. 4, pp. 2563-2572, 2010.

[13] X. Li and H. Wang, "Homoclinic and heteroclinic orbits and bifurcations of a new Lorenz-type system," International Journal of Bifurcation and Chaos, vol. 21, no. 9, pp. 2695-2712, 2011.

[14] Q. Yang, G. Chen, and T. Zhou, "A unified Lorenz-type system and its canonical form," International Journal of Bifurcation and Chaos, vol. 16, no. 10, pp. 2855-2871, 2006.

[15] O. E. Rössler, "An equation for continuous chaos," Physics Letters A, vol. 57, no. 5, pp. 397-398, 1976.

[16] J. C. Sprott, "Some simple chaotic flows," Physical Review E, vol. 50, no. 2, pp. R647-R650, 1994.

[17] Q. Yang and G. Chen, "A chaotic system with one saddle and two stable node-foci," International Journal of Bifurcation and Chaos, vol. 18, no. 5, pp. 1393-1414, 2008.

[18] J. C. Sprott, Elegant Chaos Algebraically Simple Chaotic Flows, World Scientific Publishing, Toh Tuck Link, Singapore, 2010.

[19] X. Wang and G. Chen, "A chaotic system with only one stable equilibrium," Communications in Nonlinear Science and Numerical Simulation, vol. 17, no. 3, pp. 1264-1272, 2012.

[20] C. Sparrow, The Lorenz Equations: Bifurcation Chaos, and Strange Attractor, Springer-Verlag, NewYork, NY, USA, 1982.

[21] T. Uate and G. R. Chen, "'Bifurcation analysis of Chen's equation," International Journal of Bifurcation and Chaos, vol. 10, no. 8, pp. 1917-1931, 2000.

[22] X. Y. Li and P. Wang, "Hopf bifurcation and heteroclinic orbit in a 3D autonomous chaotic system," Nonlinear Dynamics, vol. 73, no. 1-2, pp. 621-632, 2013.

[23] H. J. Wang and X. Y. Li, "More dynamical properties revealed from a 3D Lorenz-like system," International Journal of Bifurcation and Chaos, vol. 24, no. 10, 2014.

[24] H. J. Wang and X. Y. Li, "Mathematical methods in the applied sciences," Mathematical Methods in the Applied Sciences, vol. 39, no. 7, pp. 1747-1764, 2016.

[25] H. J. Wang and X. Y. Li, "On singular orbits and a given conjecture for a 3D Lorenz-like system," Nonlinear Dynamics, vol. 80, no. 1-2, pp. 969-981, 2015.

[26] H. J. Wang and X. Y. Li, "Infinitely many heteroclinic orbits of a complex Lorenz system," International Journal of Bifurcation and Chaos, vol. 27, no. 7, 2017.

[27] T. Zhou, Y. Tang, and G. Chen, "Complex dynamical behaviors of the chaotic Chen's system," International Journal of Bifurcation and Chaos, vol. 13, no. 9, pp. 2561-2574, 2003.

[28] P. Yu, M. A. Han, and Y. Z. Bai, "Dynamics and bifurcation study on an extended Lorenz system," Journal of Nonlinear Modeling and Analysis, vol. 1, no. 1, pp. 107-128, 2019.

[29] C. X. Liu, L. Liu Ling, T. Liu, and P. Li, "A new butterfly-shaped attractor of Lorenz-like system," Chaos, Solitons and Fractals, vol. 28, pp. 1196-1203, 2006.

[30] X. Li, H. J. Wang, and H. Wang, "A three-dimensional nonlinear system with a single heteroclinic trajectory," Journal of Applied Analysis \& Computation, vol. 10, no. 1, pp. 249-266, 2020.

[31] Y. A. Kuzenetsov, Elements of Applied Bifurcation Theory, Springer-Verlag, New York, NY, USA, 3rd edition, 2004.

[32] A. Cima and J. Llibre, "Bounded polynomial vector fields," Transactions of the American Mathematical Society, vol. 318, no. 2, pp. 557-579, 1990.

[33] M. Messias, "Dynamics at infinity and the existence of singularly degenerate heteroclinic cycles in the Lorenz system," Journal of Physics A: Mathematical and Theoretical, vol. 42, Article ID 115101, 2009. 